OPEN ACCESS

Edited by:

José M. Romero,

University of Seville, Spain

Reviewed by:

Nigel G. Halford

Rothamsted Research (BBSRC), UK Jianchang Du,

Jiangsu Academy of Agricultural Sciences, China

${ }^{*}$ Correspondence:

Xilin Hou

hxl@njau.edu.cn

Specialty section:

This article was submitted to Plant Evolution and Development,

a section of the journal

Frontiers in Plant Science

Received: 28 October 2016

Accepted: 25 January 2017

Published: 10 February 2017

Citation:

Wu P, Wang W, Duan W, Li Y and Hou X (2017) Comprehensive Analysis of the CDPK-SnRK Superfamily Genes in Chinese Cabbage and Its Evolutionary Implications in Plants.

Front. Plant Sci. 8:162.

doi: 10.3389/fp/s.2017.00162

\section{Comprehensive Analysis of the CDPK-SnRK Superfamily Genes in Chinese Cabbage and Its Evolutionary Implications in Plants}

\author{
Peng $\mathrm{Wu}^{1}$, Wenli Wang ${ }^{1}$, Weike Duan ${ }^{1,2}$, Ying $\mathrm{Li}^{1}$ and Xilin Hou ${ }^{1 *}$ \\ ${ }^{1}$ State Key Laboratory of Crop Genetics and Germplasm Enhancement, Key Laboratory of Biology and Germplasm \\ Enhancement of Horticultural Crops in East China, Ministry of Agriculture, Nanjing Agricultural University, Nanjing, China, \\ ${ }^{2}$ School of Life Science and Food Engineering, Huaiyin Institute of Technology, Huaian, China
}

The CDPK-SnRK (calcium-dependent protein kinase/Snf1-related protein kinase) gene superfamily plays important roles in signaling pathways for disease resistance and various stress responses, as indicated by emerging evidence. In this study, we constructed comparative analyses of gene structure, retention, expansion, whole-genome duplication (WGD) and expression patterns of CDPK-SnRK genes in Brassica rapa and their evolution in plants. A total of 49 BrCPKs, 14 BrCRKs, 3 BrPPCKs, 5 BrPEPRKs, and $56 \mathrm{BrSnRK}$ s were identified in B. rapa. All BrCDPK-SnRK proteins had highly conserved kinase domains. By statistical analysis of the number of CDPK-SnRK genes in each species, we found that the expansion of the CDPK-SnRK gene family started from angiosperms. Segmental duplication played a predominant role in CDPK-SnRK gene expansion. The analysis showed that PEPRK was more preferentially retained than other subfamilies and that CPK was retained similarly to SnRK. Among the CPKs and SnRKs, CPKIII and SnRK1 genes were more preferentially retained than other groups. CRK was closest to CPK, which may share a common evolutionary origin. In addition, we identified 196 CPK genes and 252 SnRK genes in 6 species, and their different expansion and evolution types were discovered. Furthermore, the expression of BrCDPK-SnRK genes is dynamic in different tissues as well as in response to abiotic stresses, demonstrating their important roles in development in B. rapa. In summary, this study provides genome-wide insight into the evolutionary history and mechanisms of CDPK-SnRK genes following whole-genome triplication in B. rapa.

Keywords: CDPK-SnRK genes, Brassica rapa, evolutionary conservation, synteny analysis, evolutionary pattern, expression pattern

\section{INTRODUCTION}

Plants are remarkably responsive to a variety of environmental stimuli, including pathogen attack, wounding, cold, drought reception, and fluctuations in incident light (Kudla et al., 2010). Meanwhile, a variety of internal substances also affect plants growth. These external and internal signals compose a complex regulatory network that allows plants to develop in balance. Following the detection of a stress stimulus, various signal transduction pathways are switched on, resulting 
in physiological changes in the plant cell. As second messengers, calcium ions play an essential role in many important cellular processes, especially under stress conditions (Trewavas and Malhó, 1998; Sanders et al., 1999; Berridge et al., 2000). In plants, transient changes in calcium content in the cytosol (calcium signatures) have been observed during growth, development and stress conditions (Evans et al., 2001; Harper, 2001; Knight and Knight, 2001; Sanders et al., 2002). Intracellular $\mathrm{Ca}^{2+}$ signals are produced in plant cells by a variety of stimuli, such as changes in environmental conditions, interaction with microbes, and developmental programs (Bush, 1995; Ehrhardt et al., 1996; Hammond-Kosack and Jones, 1996; Knight et al., 1996; Taylor and Hepler, 1997; Pei et al., 2000; Assmann and Wang, 2001; Murata et al., 2001; McAinsh et al., 2002; Plieth and Trewavas, 2002; Ritchie et al., 2002). Plants have multiple calcium stores, including the apoplast, vacuole, nuclear envelope, endoplasmic reticulum (ER), mitochondria and chloroplasts. Therefore, each stimulus can elicit a characteristic $\mathrm{Ca}^{2+}$ wave by specifically altering the activity of various differentially localized $\mathrm{Ca}^{2+}$ channels, $\mathrm{H} / \mathrm{Ca}^{2+}$ antiporters, and $\mathrm{Ca}^{2+}$ and $\mathrm{H}^{+}$ATPases (Thuleau et al., 1998; Allen et al., 2000; Harmon et al., 2000; Hwang et al., 2000). Different calcium sensors recognize specific calcium signatures and transduce them into downstream effects, including altered protein phosphorylation and gene expression patterns (Sanders et al., 1999; Rudd and Franklin-Tong, 2001).

In eukaryotes, calcium-dependent protein kinases (CDPKs) and most sucrose non-fermenting-1-related kinases (SnRKs) are involved in regulating and decoding $\mathrm{Ca}^{2+}$ signals (Assmann and Wang, 2001; Evans et al., 2001; Harmon et al., 2001; Cheng et al., 2002; Fasano et al., 2002; Hrabak et al., 2003; Cho et al., 2009; Kulik et al., 2011). The protein kinases also involved in stress signal transduction in plants are common to all eukaryotic organisms and include mitogen-activated protein kinases (MAPKs), glycogen synthase kinase 3 (GSK3), and S6 kinase (S6K). The CDPK-SnRK superfamily consists of seven types of protein kinases, which differ in the regulatory domains they contain (Harmon et al., 2001). CDPKs (also named CPKs) are activated by the binding of calcium to their calmodulinlike regulatory domains. The carboxyl terminal domains of CRKs (CDPK-related kinases) have sequence similarity to the regulatory domains of CPKs but do not bind calcium. PEPRKs (PEP carboxylase kinases) contain only one catalytic domain (Harmon et al., 2001). PPCKs (PEPC kinases) have a carboxyl-terminal domain that has no similarity to that of any other member of the superfamily (Hrabak et al., 2003). CCaMKs (calcium- and calmodulin-dependent protein kinases) bind both calcium ions and the calcium/calmodulin complex, whereas CaMKs (calmodulin-dependent protein kinases) bind the calcium/calmodulin complex but not calcium (Hrabak et al., 2003). In addition, there are the classical SNF1-type kinases from yeast; Halford and Hardie (1998) proposed the name SNF1-related kinase (SnRK) for this group and recognized three subgroups: SnRK1, SnRK2, and SnRK3 (Harmon et al., 2001). However, CaMK and CCaMK are absent from Arabidopsis (Hrabak et al., 2003). All members of the CDPK-SnRK gene superfamily have kinase domains of similar length and sequence and a similar general organization, with the kinase domains at or near the N-terminus, then the junction domains, followed by the regulatory domains (Harmon, 2003; Hrabak et al., 2003).

The plant CPKs characterized to date play substantive roles in diverse physiological processes. These processes include tolerance to salt, cold, and drought stress in rice (Saijo et al., 2000), the defense response in tobacco (Romeis et al., 2000), the accumulation of storage starch and protein in immature seeds of rice (Asano et al., 2002), the regulation and development of nodule number in Medicago truncatula (Gargantini et al., 2006), and the response to ABA in Arabidopsis (Choi et al., 2005) (38). The original, systematic report on the CPK genes family in Arabidopsis thaliana identified 34 CPK genes family members (Choi et al., 2005) and was followed by research in rice (Oryza sativa) (Ray et al., 2007) and wheat (Triticum aestivum) (Li et al., 2008). Recently, genome-wide analyses of the CPK gene family have been reported in maize (Zea mays) (Kong et al., 2013) and poplar (Populus trichocarpa) (Zuo et al., 2013). Meanwhile, more and more investigations of CPK genes have also involved horticultural plants, such as alfalfa (Davletova et al., 2001), potato (Raíces et al., 2003), strawberry (Llop-Tous et al., 2002), and tomato (Chico et al., 2002). Furthermore, research using transgenic plants has revealed the biological functions of a few CPK genes in higher plants. Transgenic rice constitutively overexpressing OsCPK7 or OsCPK13 showed enhanced tolerance to cold, salt, and drought stress (Saijo et al., 2000; Komatsu et al., 2007). In tobacco, CPK-silenced plants displayed a reduced and delayed hypersensitive response to the fungal Avr9 elicitor (Romeis et al., 2001). GhCPK1 was the first cotton CPK gene to be identified and was considered to play a role in the calcium signaling events associated with fiber elongation (Huang et al., 2008). Arabidopsis thaliana CPK23 (AtCPK23) is a positive regulator of the response to drought and salt stress ( $\mathrm{Ma}$ and $\mathrm{Wu}$, 2007), whereas AtCPK6 may be crucial in positively regulating methyl-jasmonate signaling in guard cells (Munemasa et al., 2011). In addition, the overexpression of rice (Oryza sativa) CPK7 (OsCPK7) significantly improves resistance to cold (Komatsu et al., 2007). Phytohormones are involved in the responses to abiotic stresses; therefore, the expression levels of members of the CPK gene family has also been shown to be regulated after treatment with various phytohormones, such as $\mathrm{ABA}$, auxin and jasmonic acid. Recently, Zea mays CPK11 was reported as a component of the jasmonic acid signaling pathway, and its concentration in cells was observed to increase in response to wounding and touch (Szczegielniak et al., 2012).

Sucrose non-fermenting-1-related protein kinase (SnRK) is homologous to SNF1 and AMP-activated protein kinase (AMPK), which is widely distributed in plants and is involved in a variety of signaling pathways. SnRK is the key switch in plant sugar signaling, stress, seed germination and seedling growth. SNF1 of yeast, AMPK of mammals and SnRK1 of plants are homologous, belonging to the SNF1 protein kinase superfamily. SNF1 was found in yeast (Saccharomyces cerevisiae) originally (Alderson et al., 1991). In yeast, glucose regulates the protein-protein interaction, substrate specificity and subcellular localization of the SNF1 subunit that modulates SNF1 kinase activity, resulting in the phosphorylation of activators and repressors that control transcription of multiple genes in 
metabolic pathways required for the utilization of alternative energy sources. In the eukaryote, SNF1 protein kinase is very strongly conserved. Many SNF1 analogs have been identified in plants. SnRK1 was discovered initially in rye (Secale cereale L.) (Alderson et al., 1991). At present, some members of the SnRK1 subfamily have been found in variety of model plants and some important crops, such as Arabidopsis thaliana, rye, barley (Hordeum vulgare), potatoes (Solanum tuberosum), tobacco, beets, etc. It may exist in all plants (Halford and Hardie, 1998; Halford et al., 2003). Studies have shown that SnRK1 is the key switch in plant sugar signaling. In addition, the regulation of glucose metabolism, hormonal regulation and sugar signaling is directly related to signal transduction (Kleinow et al., 2000; Jossier et al., 2009; Mathieu et al., 2009). SnRK2s are a plantspecific Ser/Thr protein kinase family. All of the members have a conserved $\mathrm{N}$-terminal catalytic domain similar to that of SNF1/AMPK-type kinases and a short C-terminal regulatory domain that is not highly conserved. Prior to 2000, there were only a small number of studies indicating that ABA and abiotic stresses induced the expression of some SnRK2 genes (Anderberg and Walker-Simmons, 1992; Holappa and WalkerSimmons, 1995). In 2000, SnRK2s began to be recognized as enzymes involved in abiotic stress signal transduction in plants (Li et al., 2000). By 2003, 10 SnRK2 genes had been identified and were renamed SnRK2.1 through SnRK2.10 (Hrabak et al., 2003). In 2009, independently, two laboratories obtained a triple SnRK2.2/2.3/2.6 mutant. SnRK2.2/2.3/2.6 triple-mutant plants are nearly completely insensitive to $\mathrm{ABA}$, which was used to establish the role of ABA-dependent SnRK2s in the plant response to water deficit, seed maturation, and germination. These reports indicate that $S n R K 2.2 / 2.3 / 2.6$ function as primary positive regulators and suggest that ABA signaling is controlled by the dual modulation of SnRK2.2/3/6 and group A PP2Cs (Fujii and Zhu, 2009; Fujii et al., 2009; Nakashima et al., 2009). SnRK3 is a protein kinase in plants, called calcineurin B-like calcium sensor-interacting protein kinase (CIPK) (Kim et al., 2000). CIPK interacts with the calcium-binding protein SOS3, SCaBPS and CBL (calcineurin B-like calcium sensor). Studies have shown that CIPK and an upstream complex of CSL interactions are involved in salt stress, sucrose and ABA signal transduction (Imamura et al., 2008). In Arabidopsis, PKS3, PKS18 and CIPK3 of the SnRK3 family can regulate plant growth, stomatal opening and closing and seed germination under ABA treatment (Kim et al., 2003). Arabidopsis AtCIPK1 forms complexes with AtCBL1 and AtCBL9, regulating ABA-independent and ABA-dependent pathways, respectively (D'Angelo et al., 2006). AtCIPK3 regulates ABA and cold signal transduction pathways (Kim et al., 2003). Girdhar's study showed that CBL9 interacted with CIPK3 to regulate the $\mathrm{ABA}$ pathway, and this finding was validated in a yeast two-hybrid experiment (Pandey et al., 2008).

During their evolution, plants have substantially altered their phenotypes to adapt to environmental changes by transforming the form and function of genes. Gene duplication, even a whole-genome duplication (WGD), offers the chance for genes to change (Rensing, 2014). Angiosperm genome evolution is characterized by polyploidization through WGD followed by diploidization, which is typically accompanied by considerable homoeologous gene loss (Stebbins, 1950). After duplication, one copy of the gene might either becomes non-functional (pseudogenized or silenced, also called gene death) or acquire a novel function (neofunctionalization). Alternatively, the two duplicates might divide the original function of the gene (Innan and Kondrashov, 2010). Preliminary analyses revealed that gene duplication and subsequent divergence are the main contributors to evolutionary momentum (Ohno et al., 1968; Chothia et al., 2003). The genome of $A$. thaliana has experienced a paleohexaploidy $(\beta)$ duplication shared with most dicots and two subsequent genome duplications ( $\alpha$ and $\gamma$ ) since its divergence from Carica papaya, along with rapid DNA sequence divergence and extensive gene loss (Bowers et al., 2003). In A. thaliana, some duplicated regions found in CDPK-SnRK protein kinases indicated that CDPK-SnRK protein kinases are paralogs that arose by divergence after genome duplication events (Hrabak et al., 2003). The CPK genes of Arabidopsis and maize have undergone both segmental and tandem duplication, contributing to the expansion of the CPK family. In Populus, however, segmental duplication played a predominant role in the expansion of CPK genes (Zuo et al., 2013). In addition, tandem duplication of CPK genes has not occurred in the rice genome (Asano et al., 2005).

In this study, we constructed a comprehensive comparative analysis of CDPK-SnRK genes, including phylogenetic relationships, gene structures, chromosome distribution, gene retentions, gene expansions, gene duplication and gene expression patterns, in different tissues to characterize the divergences in composition, expansion, and expression. First, we identified 555 CPKs, 120 CRKs, 5 PPCKs, 14 PEPRKs, and 697 SnRKs in 16 plant species. Second, we conducted a comparative genomic analysis of these genes with 16 other plant and species found that the expansion of the CDPK-SnRK family from angiosperms mainly relied on WGDs. Third, PEPRK genes were more preferentially retained than other subfamilies and CPK genes were retained similarly to SnRK genes during diploidization following WGT in B. rapa. Fourth, during the course of evolution, CPK appeared most recently and expanded most rapidly. Fifth, the expressions of CDPK-SnRK genes are dynamic in different tissues as well as in response to abiotic stresses, demonstrating their important roles in development in B. rapa. This study is the first report on CDPK-SnRK genes in $B$. rapa. and extends our understanding of the roles of the CDPK-SnRK gene superfamily in evolution and stress responses.

\section{RESULTS}

\section{Identification and Classification of the CDPK-SnRK Superfamily of Protein Kinases in Brassica rapa and Comparative Analyses}

In this study, genome-wide analysis of CDPK-SnRK gene family has been performed on the basis of the completed B. rapa genome sequence (Wang et al., 2011). Based on previously reported methods (Harmon et al., 2001; Hrabak et al., 2003), the homogeneous candidate CDPK-SnRK genes between 
Brassica rapa and other species were identified by BLASTP (Supplementary Table 1). Subsequently, all candidate protein sequences were subjected to Pfam and SMART analyses. Finally, we identified $49 \mathrm{BrCPKs}, 14 \mathrm{BrCRKs}, 3 \mathrm{BrPPCKs}, 5 \mathrm{BrPEPRKs}$, and $56 \mathrm{BrSnRKs}$ named according to nomenclature proposed for CDPK-SnRK genes (Supplementary Table 2).

To better understand the expansion and evolutionary history of CDPK-SnRK genes in B. rapa, genes were also identified in 16 other species representing the major clades of plants. The evolutionary relationships of the species and the number of CDPK-SnRK genes are shown in Figure 1A. The data show that Glycine max contained the highest number of CDPK-SnRK genes (200), followed by Z. mays (193) and M. truncatula (188) (Figure 1A). However, A. trichopoda, a basal angiosperm species that was the single living representative of the sister lineage to all other extant flowering plants, contained the lowest number of CDPK-SnRK genes (28) in Angiospermae. The reason is that it originated prior to the split of eudicots and monocots and has not experienced any whole genome duplication (WGD), while the other 12 angiosperms had several rounds of WGDs/triplications after their split from $A$. trichopoda. Furthermore, the number of CDPK-SnRK genes in algae, Bryophyta and Pteridophyta was less than that in Angiospermae. This phenomenon was also caused by several WGD events that occurred during angiosperm evolution (Figure 1B). These results indicated that the expansion of the CDPK-SnRK family from angiosperms mainly relied on large-scale DNA rearrangements, namely, WGDs. The elevated duplication frequency and increased retention of CDPK-SnRK genes also contributed to neofunctionalization and caused them to gain important functions in angiosperm development.

\section{Characteristics of Structure, and Expansion Analysis of BrCDPK-SnRK Proteins}

To investigate the extent of lineage-specific expansion of the CDPK-SnRK genes in B. rapa, phylogenetic trees were constructed using the maximum likelihood method (Figure 2A). The phylogenetic tree showed that all the CDPK-SnRK genes were clustered into five distinct gene classes (CPK, CRK, PEPRK, PPCK, SnRK) (Figure 2A), while the CPK family was divided into four groups (I, II, III, and IV) and the SnRK family was classified into three groups (SnRK1, SnRK2, and SnRK3), consistent with the reports in A. thaliana (Hrabak et al., 2003). In B. rapa, the CPK, CRK, PEPRK, PPCK, and SnRK gene families contained 49 members, 14 members, 3 members, 5 members, and 56 members, respectively, whereas in A. thaliana, the CPK, CRK, PEPRK, PPCK, and SnRK families contained 34 members, 8 members, 2 members, 2 members, and 39 members, respectively. Next, the synteny of CDPK-SnRK genes between A. thaliana and three subgenomes in B. rapa was analyzed. There were $34 C P K$, $8 C R K, 2$ PEPRK, 2 PPCK, and 38 SnRK genes on the conserved collinear block (Supplementary Table 5). Meanwhile, 2 CPKs, 2 CRKs, 1 PEPRK, 1 PPCK, and 3 SnRKs were retained completely; conversely, $4 C P K s$ and $3 S n R K s$ from $B$. rapa were lost. Due to the Brassica-specific WGT event, the gene numbers of these classes in B. rapa were greater than those in A. thaliana.
Furthermore, the different domain architectures, motif compositions and gene structures of CDPK-SnRK were analyzed (Figure 2B, Figure S1). All members of the CDPK-SnRK superfamily have a kinase domain of similar length and sequence, with the kinase domains at or near the N-terminus, then the junction domains, followed by the regulatory domains (Figure 2B). Although CPK proteins have a functional kinase domain coupled with regulatory calcium-binding EF-hands, the C-terminal domains (EF-hands) of CRK proteins contain apparently degenerate calcium-binding sites with no function. Meanwhile, 10 conserved motifs were detected in BrCPK, BrCRK, BrPEPRK, BrPPCK, and BrSnRK, respectively (Figure S3). All BrCDPK-SnRK proteins had highly conserved kinase domains, which corresponded to motifs $1-4,7,9$ in BrCDPK and motifs 1-4,6,7,9 in BrSnRK, whereas motif 8 was found in $B r S n R K 3.1$, corresponding to the NAF/FISL domains (Figures $\mathrm{S} 1 \mathrm{~A}, \mathrm{~B})$. The amino acid sequence of BrCDPK-SnRK was aligned with AtCDPK-SnRK protein sequences from five gene classes. In CPK, CRK, PEPRK, PPCK, and SnRK, higher sequence similarities were identified in the N-terminus, which corresponded to the conserved kinase domain (Figure S2). In addition, variable gene structures of BrCDPK-SnRK were observed. As shown in Figures 3A,C, the intron numbers of the BrCPK genes ranged from 5 to 9 with a median of 6 , while the BrSnRK genes ranged from 0 to 15 with a median of 7 . Interestingly, we found that $22 \mathrm{BrSnRK} 3$ genes have no introns. The theoretical pI of the BrCPK gene family ranged from 4 to 9 with a median of 6 , but BrSnRK proteins showed a $\mathrm{pI}$ range from 2 to 11 and with a median of 8 (Figure 3B). Other classes had complex theoretical pI ranging in value from 4 to 9 (Figure 3B).

\section{Different Retention of CDPK-SnRK Genes Following WGT in Brassica rapa}

To investigate different retention in CPK, CRK, PEPRK, PPCK, and SnRK during B. rapa WGT events, 44/49, 14/14, $3 / 3,5 / 5$, and $50 / 56$ were located in the syntenic regions, respectively (Figures 4A,B and Supplementary Table 3). The results demonstrated that $43 \%(44 / 102)$ of the CPK genes were retained in the syntenic regions, relative to $44 \%(50 / 114)$ of the SnRK genes. The retention rates of CRK, PEPRK and PPCK are 58\% (14/24), 50\% (3/6), and 83\% (5/6), respectively (Figure 4F). Additionally, we counted gene copies and analyzed the distribution of the three subgenomes by comparing the retention of CPK, CRK, PEPRK, PPCK, and SnRK (Figure 4E). The result showed that all PEPRK genes had more than two copies retained, which is more than the retention of the other subfamilies (42\%). However, 3\% of the CPK and SnRK genes were completely lost. Next, the proportions of $\mathrm{CPK}$ and SnRK genes retained were higher in the least fractionated (LF) subgenome than in the medium fractionated (MF1) and most fractionated (MF2) subgenome, consistent with a previous report showing that the degree of retained genes in these three subgenomes (LF, MF1, and MF2) was decreased (Wang et al., 2011). In contrast, the PEPRK and PPCK families were retained more in the MF1 subgenome 

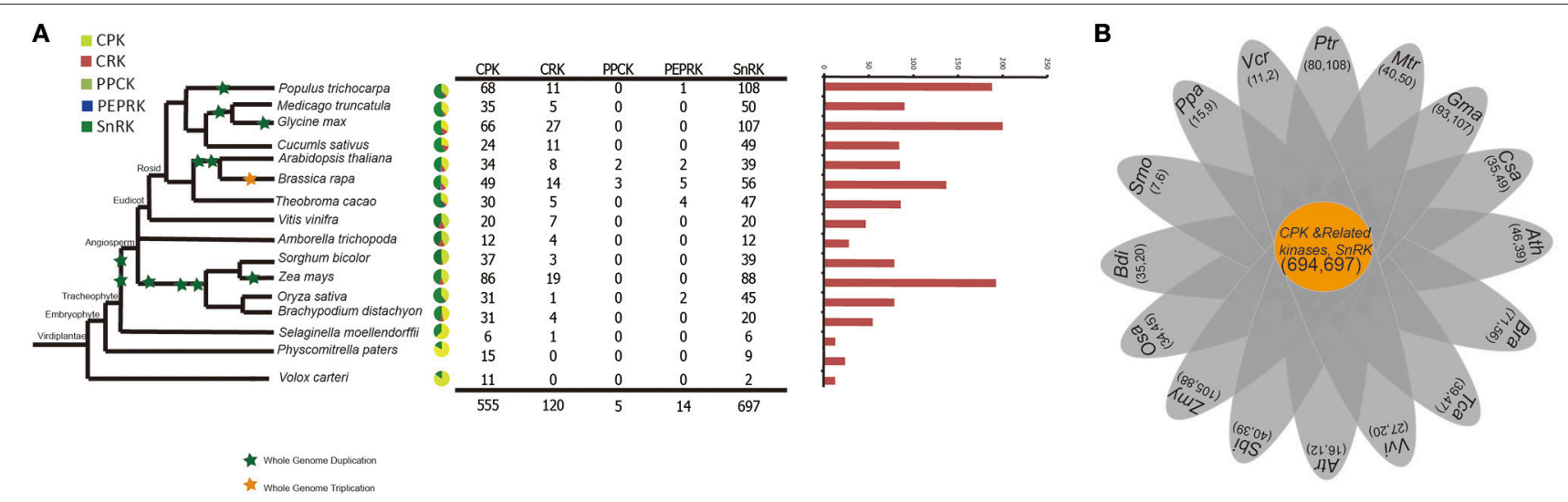

FIGURE 1 | A comparative analysis of CDPK-SnRK genes in plants. (A) The evolutionary relationships and the numerical details of the CDPK-SnRK superfamily of each species. (B) The Venn diagram shows the number of common gene families and genes in 16 plants.

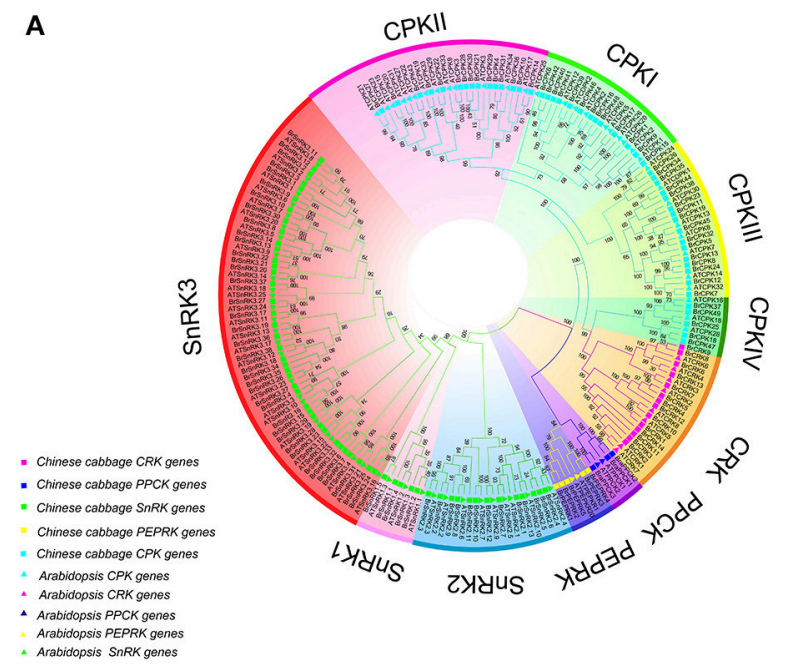

B

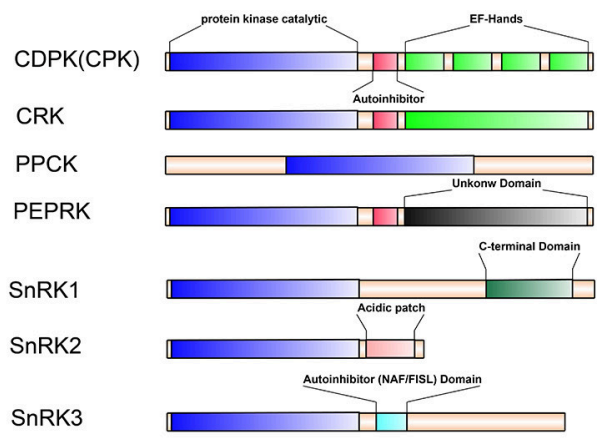

FIGURE 2 | Phylogenetic tree and major domains of CDPK-SnRK proteins. (A) Phylogenetic analysis of 212 CDPK-SnRK proteins from Chinese cabbage (127) and Arabidopsis (85) showing similar groups in all of the plant species. In total, 10 clades with different colors that were formed by CDPK-SnRK proteins are also marked. (B) The kinases are drawn with the N-terminus to the left and C-terminus to the right. Catalytic domains (blue boxes) have been aligned to facilitate comparison. Auto-inhibitory domains are shown as red boxes. EF-hands are shown as green boxes within the regulatory domains. The SnRK1 C-terminus domains are shown as dark green boxes. Acidic patch domains are shown as pink boxes. The SnRK3 NAF/FISL domains are shown as dark and light blue boxes.

than in the LF subgenome (Figure $4 \mathrm{~F}$ ). In summary, the results confirmed that PEPRK genes were more preferentially retained than other subfamilies and that CPK genes were retained similarly to SnRK genes during diploidization following WGT in B. rapa.

Furthermore, the retention rates of four CPK (CPKI, II, III, $I V)$ and three $\operatorname{SnRK}(\operatorname{SnRK1}, 2,3)$ groups were observed. As show in Figure 4H, 67\% of CPKIIIs and SnRK1s had more than two copies retained, which is greater than the retention of the other groups (Figures $4 \mathbf{H}, \mathbf{D}$ ). In addition, the proportion of CPKIIIs and SnRK1s retained was higher in the LF subgenome than in the other subgenomes, which once again confirmed that CPKIII and SnRK1 genes were more preferentially retained than other groups (Figures 4C,G).

\section{Chromosome Distribution, Ks and Duplication Analysis of the CDPK-SnRK Genes in B. rapa}

All BrCDPK-SnRK genes could be mapped onto 10 chromosomes of Chinese cabbage with a non-random distribution, except BrPPCK3, which is located in Scaffold000191 (Figure 5). On every chromosome, the proportion of $\mathrm{BrCPK}$ genes was similar to that of BrSnRK genes. However, Chromosome 09 contained more BrCPK genes (11 genes) than BrSnRK genes, whereas chromosomes 01 had the opposite. B. rapa shares two WGDs (WGD: $\alpha$ and $\beta$ ) and one wholegenome triplication event (WGT: $\gamma$ ) in its evolutionary history with Arabidopsis but has undergone an additional WGT event. Therefore, the B. rapa genome was further divided into three 


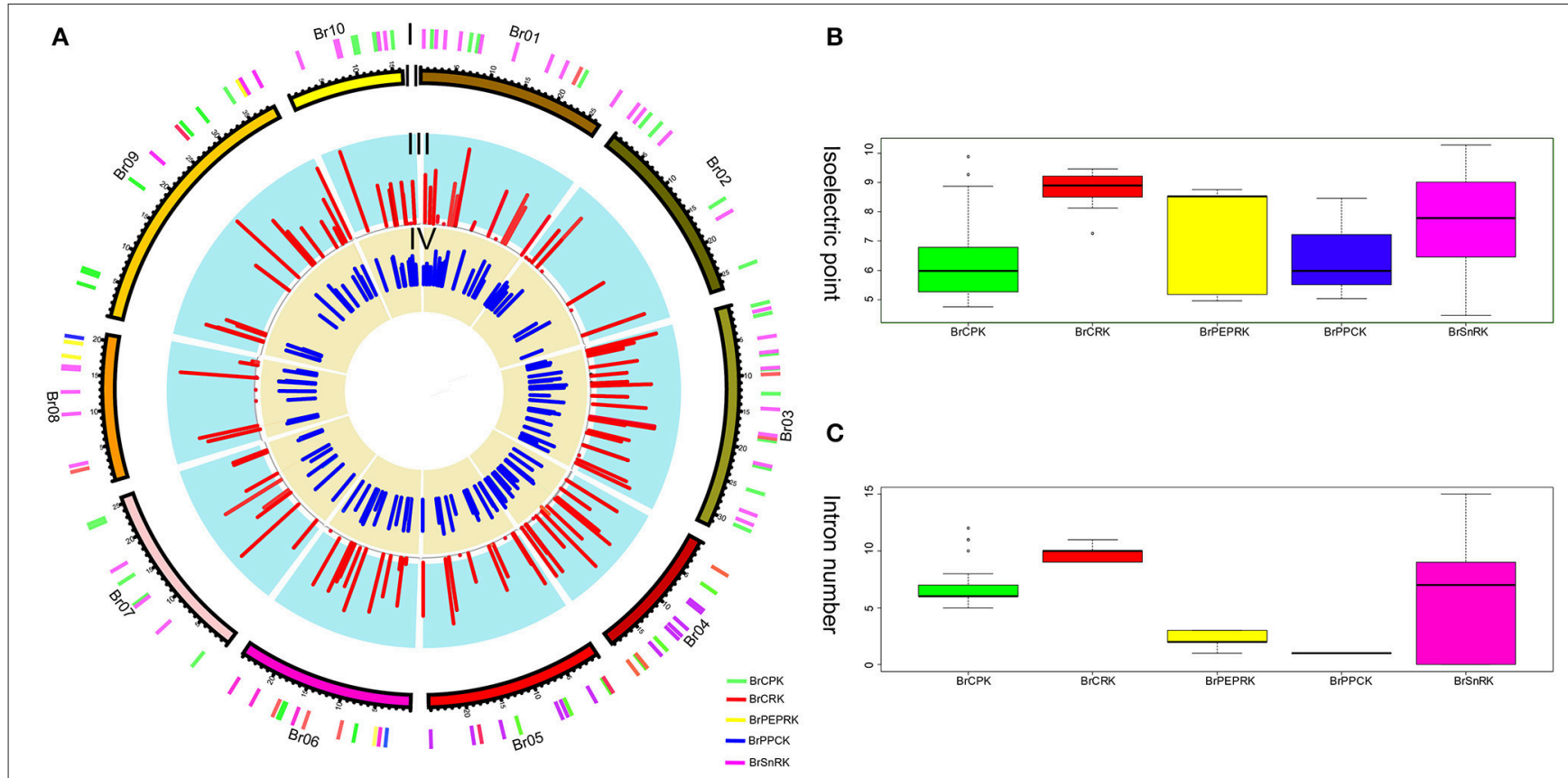

FIGURE 3 | Characteristics of isoelectric points and introns among BrCDPK-SnRK. (A) I indicates CDPK-SnRK genes within the B. rapa Genome. II indicates the B. rapa chromosome karyotype. III represents the number of introns (red) of CDPK-SnRK genes. IV indicates the Pi value (blue) of BrCDPK-SnRK genes. (B) The Pi value among gene classes BrCPK, BrCRK, BrPEPRK, BrPPCK, and BrSnRK. (C) The number of introns among gene classes BrCPK, BrCRK, BrPEPRK, BrPPCK, and BrSnRK.

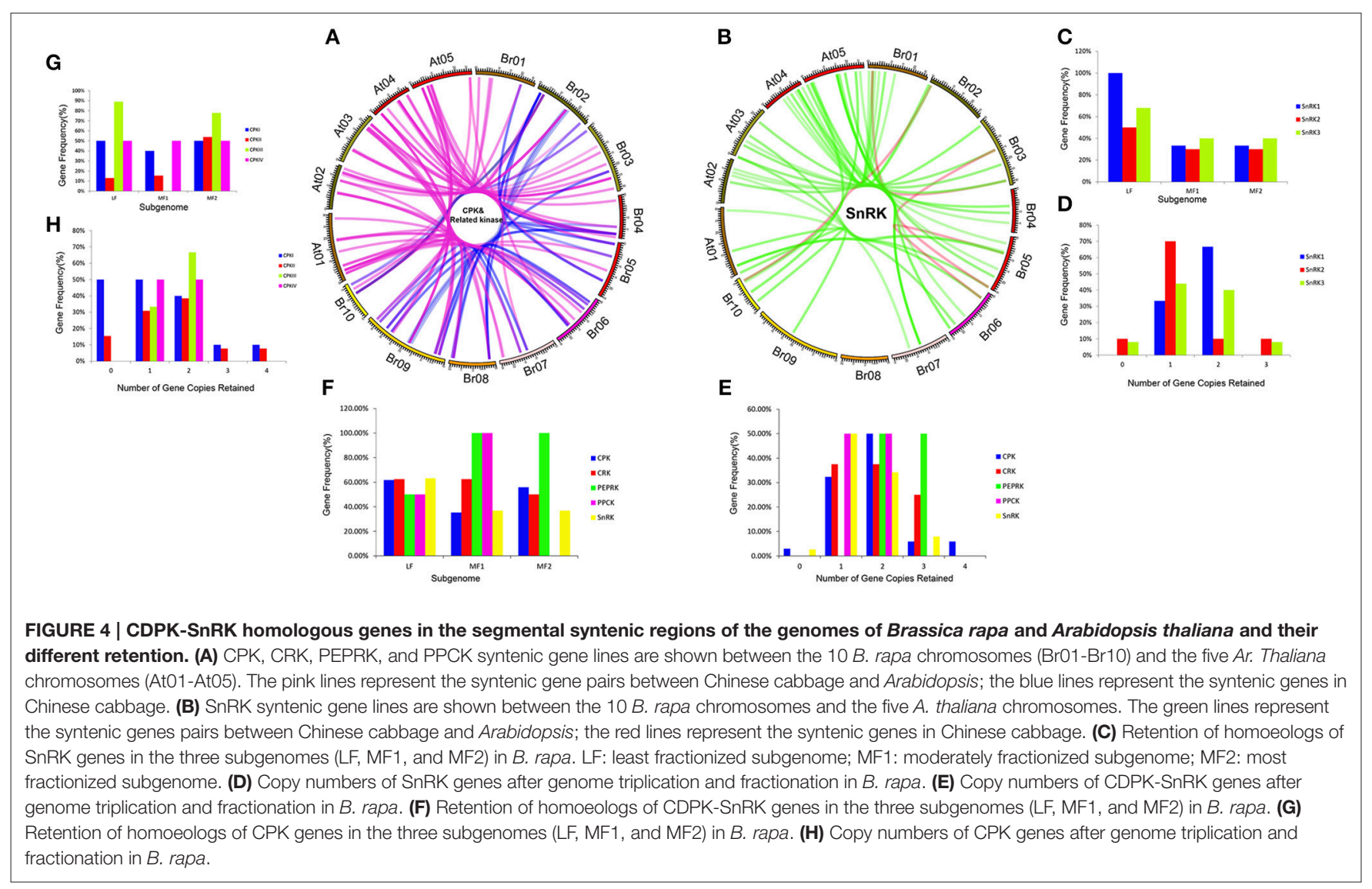




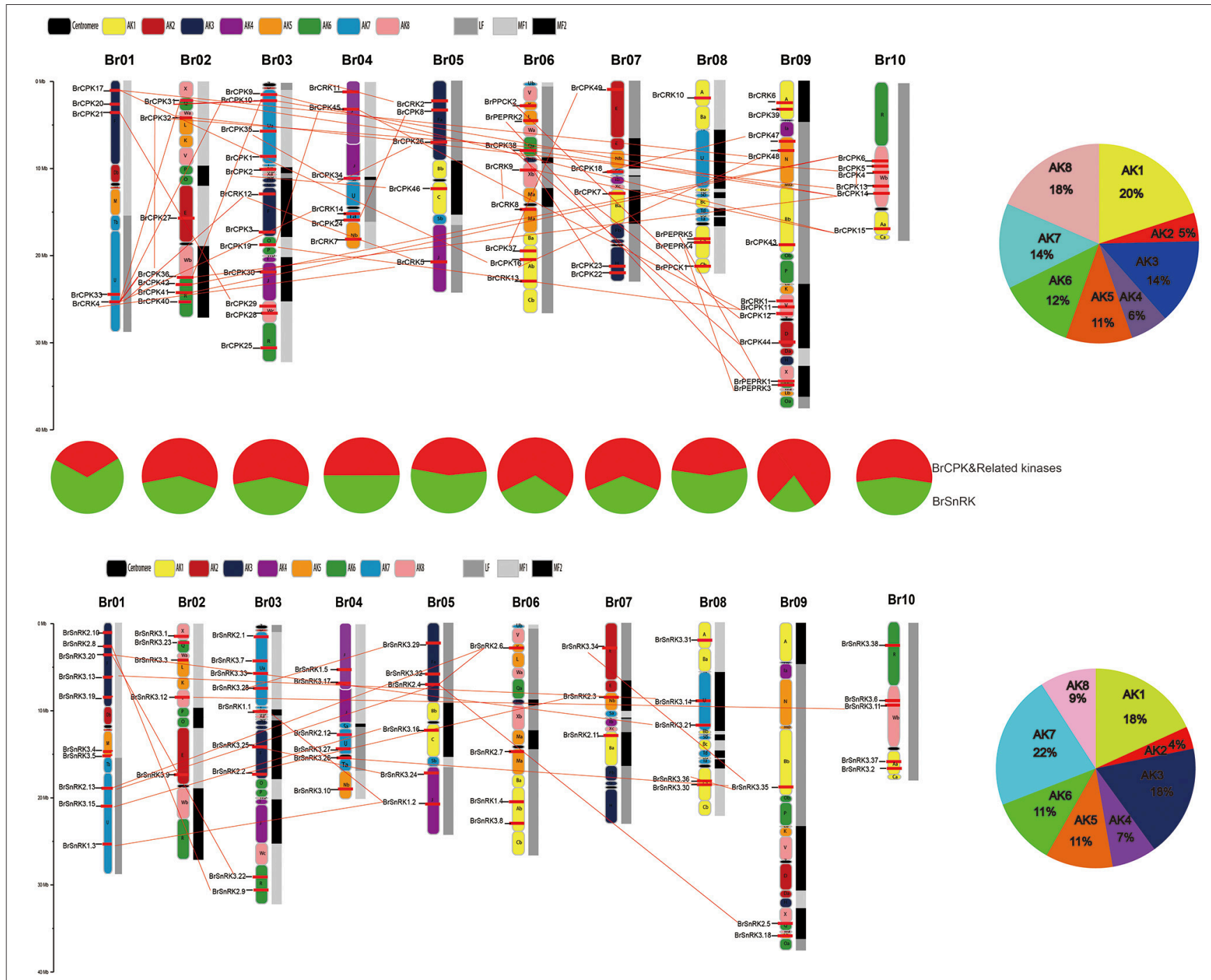

FIGURE 5 | The features of the CDPK-SnRK genes in B. rapa. The distribution of BrCDPK-SnRK genes on 10 chromosomes. The 8 ancestral blocks and three subgenomes, including the least fractionated (LF), medium fractionated (MF1), and most fractionated (MF2) subgenomes, were plotted as described by Cheng et al. (2013). AK represents the ancestral karyotype. The red lines connect the duplicated BrCDPK-SnRK genes.

differentially fractionated subgenomes (LF, MF1, MF2), of which LF contained more BrCPK genes and BrSnRK genes than either of the other two subgenomes. In addition, the 24 conserved ancestral genomic blocks (labeled $\mathrm{A}-\mathrm{X}$ ) in the $B$. rapa genome were reconstructed according to previous reports (Cheng et al., 2013). The color coding of these blocks was based on their positions in a proposed ancestral karyotype (AK1-8). We also found that most of the $\mathrm{BrCPK}$ genes cluster together in a region of AK1 (20\%), whereas BrSnRK genes belonged to AK1 (18\%) and AK3 (18\%).

Furthermore, the types were identified by the MCScanX program, and the divergence times of the duplicated genes were estimated by calculating the synonymous substitution rates $(K s)$ and non-synonymous substitution rates $(K a)$. In total, $70 \mathrm{BrCDPK}-\operatorname{SnRK}$ duplicated gene pairs were analyzed
(Supplementary Table 4). BrCPK (74\%), BrCRK (86\%), BrPEPRK (100\%), BrPPCK (100\%), and BrSnRK (70\%) duplicated gene pairs were segmental duplications (Figure 6B), and all the duplicated $B r C D P K-S n R K$ gene pairs had $\mathrm{Ka} / \mathrm{Ks}$ ratios less than 1 , indicating the purifying selection of these genes (Figure 6A, Supplementary Table 4). To understand the divergence time, the $K s$ values of the BrCPK genes ranged from 0.3 to 0.5 and had a mean of $\sim 0.34(\sim 11 \mathrm{Myr})$, while the BrSnRK genes ranged from 0.2 to 0.55 and focused on $\sim 0.25$ ( $\sim 8.5 \mathrm{Myr}$; Figures 2A,B, 6A). The divergence time of BrSnRK duplicated gene pairs was 8.49 MYA, which indicates that their divergence occurred during the Brassica triplication events ( $\sim 9$ MYA). The divergence times obtained for the BrCPK duplicated gene pairs ranged from 10 to 16.6 MYA, indicating that these duplications occurred during the divergence of Chinese cabbage and Arabidopsis (9.6-16.1 MYA). 


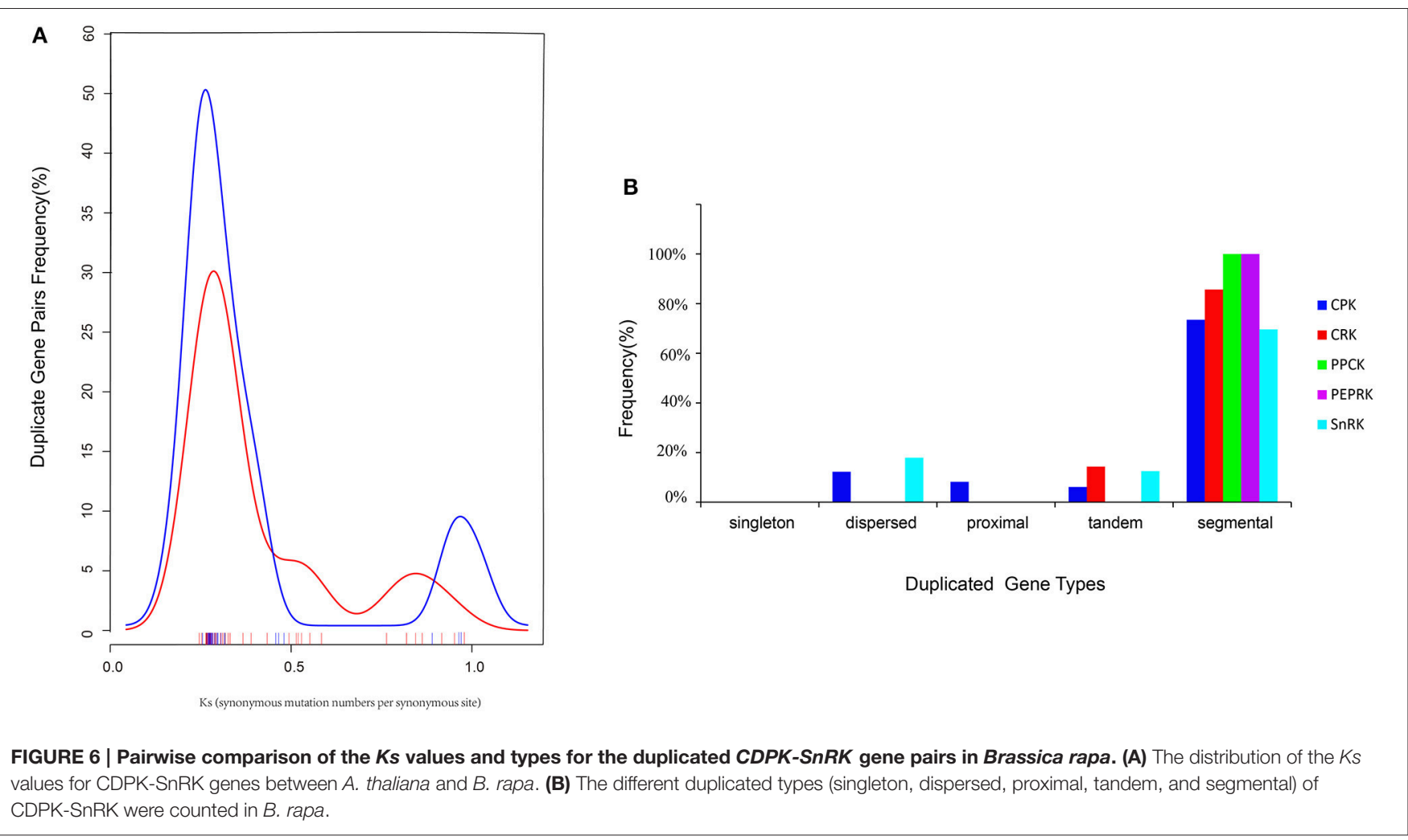

\section{Evolution Pattern of CDPK-SnRK Genes in Plants}

To investigate the evolution of the CDPK-SnRK family in the plant kingdom, we selected 13 Angiospermae (8 eudicots, 4 monocots and one basal angiosperm), 3 Gymnospermae, 1 Pteridophyta, 1 Bryophyta, and 1 Chlorophyta species for comparative analysis (Figure S3). We constructed a phylogenetic tree of the CDPK-SnRK genes to analyze the evolutionary relationships of these species. The phylogenetic tree showed that the CDPK-SnRK genes formed five gene classes (CPK, CRK, PEPRK, PPCK, and SnRK), which is consistent with the result for B. rapa and A. thaliana. Meanwhile, we found that no CRK, PEPRK, or PPCK genes were detected in Volvox carteri. Therefore, the CRK, PEPRK, and PPCK gene families may only exist in land plants. To further determine the relationship among the five groups, the genetic distance was analyzed (Figures $\mathrm{S} 4 \mathrm{~A}, \mathrm{~B})$. The box plot shows the genetic distance of CPK vs. CRK, PEPRK, and PPCK, which was smaller than SnRK vs. these groups (Figure S4B). Notably, the genetic distance between CPK and CRK was smaller than that between CPK and PEPRK or CPK and PPCK. These results indicate that CPK has a closer relationship with CRK, PEPRK, and PPCK, especially the CRK closest to $\mathrm{CPK}$, which is consistent with previous reports that plant CPK and CRK may share a common evolutionary origin (Hrabak et al., 2003).

To further investigated the footprint of the CPK CRK, PPCK, PEPRK, and SnRK families, we selected four angiosperms (C. papaya, P. trichocarpa, A. trichopoda, and V. vinifera). The reason is that Vitis vinifera, P. trichocarpa, and C. papaya did not undergo $\alpha$ and $\beta$ duplications and A. trichopoda, a basal angiosperm, did not undergo the $\gamma$ duplication event (Jiao et al., 2011b; Albert et al., 2013; Lee et al., 2013). Phylogenetic trees in each species were constructed (Figures S5, S6). In each species, the CPK family was divided into four clades (CPKI, CPKII, CPKIII, and CPKIV), and SnRK was divided into three clades (SnRK1, SnRK2, and SnRK3). CPK, CRK, and SnRK were found to exist in Amborella trichopoda, which indicates that these three groups originated from duplication events prior to the $\gamma$ event. However, PEPRK appeared between the salicoid duplication and the $\gamma$ event. The PPCK family exists in $P$. trichocarpa, which indicates that it originated from duplication events prior to the salicoid duplication. Furthermore, due to the salicoid duplication and Brassica-specific WGT events, there were more CDPK-SnRK gene family members in P. trichocarpa and B. rapa than in other species (Tuskan et al., 2006; Wang et al., 2011). In general, during the course of evolution, CPK appeared most recently and expanded most rapidly. Above all, we inferred a possible evolutionary footprint of the CPK family (Figure S7).

The family size and the percentage of CPKs in five plants suggested that CPKs expanded rapidly during evolution and further expanded in the Brassicaceae (Figure 7). WGD is known to have important impacts on the expansion and evolution of gene families in plant genomes. However, along with the gradual increase in the CPK percentage, the genes of group III were completely lost in $V$. vinifera (Figure 7C). Compared with other groups, the expansion of group III was more unstable. To further investigate the relationship among the four groups, 


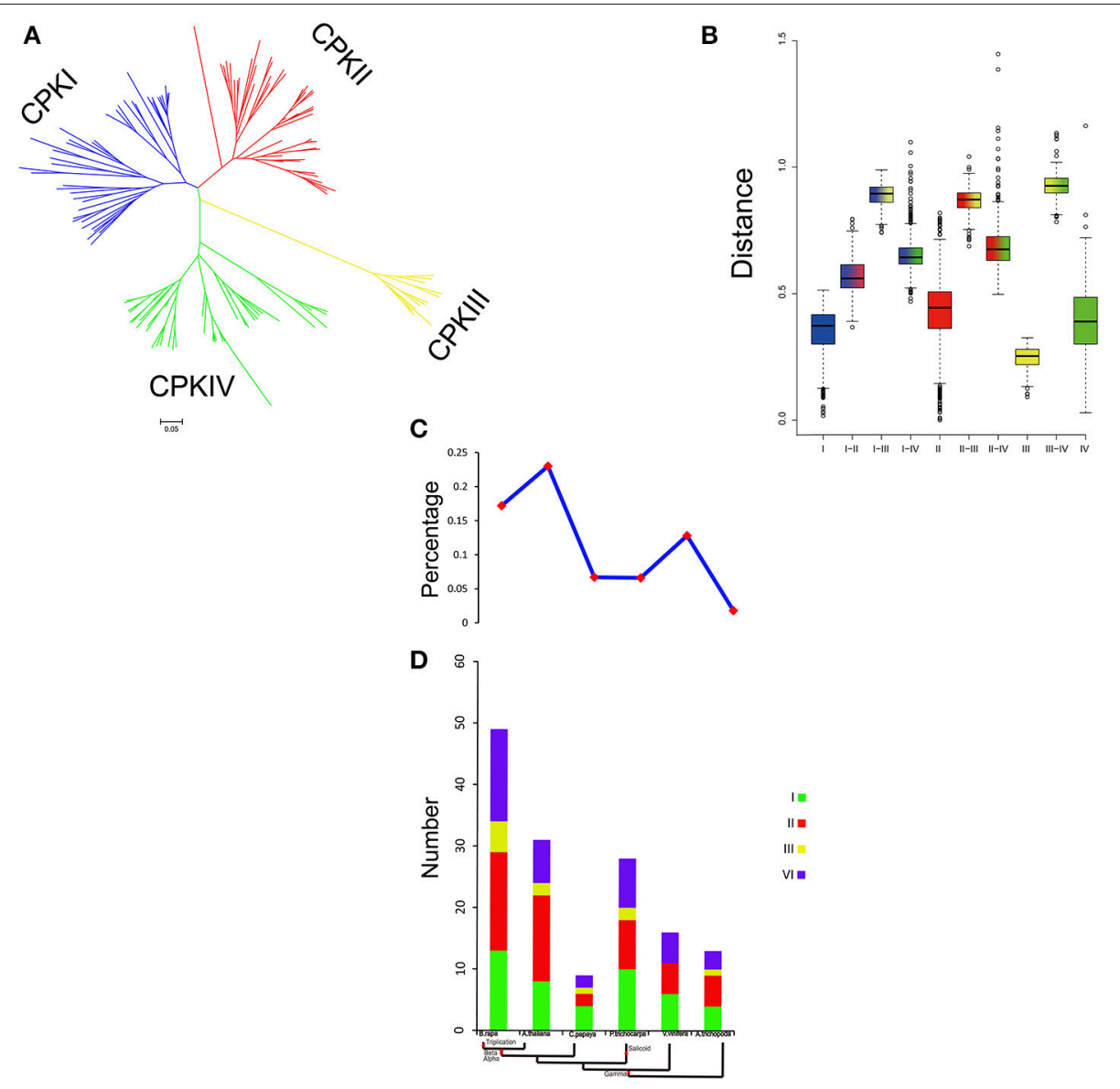

FIGURE 7 | Analysis of CPK gene evolution. (A) Phylogenetic relationships among CPK genes; (B) genetic distance among the different groups of CPK genes; (C,D) comparison of the percentage of CPK genes and copy numbers of CPK genes; CPK genes in representative species.

the genetic distance was analyzed. The results indicated that the genetic distance between CPKI and CPKIII was shorter than that between CPKI and CPKIV or that between CPKI and CPKII, and the genetic distance between CPKII and CPKIV was shorter than that between CPKII and CPKIII (Figure 7B). In summary, we inferred a possible evolutionary footprint of the CPK family. Before the $\gamma$ event, all groups in the family (CPKI, CPKII, CPKII, CPKIV) had already appeared. The gene family further expanded within Brassicaceae. Thus, the CPK family almost doubled in size in the B. rapa genome compared with that of $A$. trichopoda through three duplications, one triplication, and fractionation. The expansion of groups I, II, and IV played a major role in the expansion of the CPK gene family.

\section{Comparative Expression Pattern Analysis of the CDPK-SnRK Genes in Different Tissues of Brassica rapa and Arabidopsis thaliana}

To investigate the divergence expression profiles of CDPKSnRK genes in different organs between $A$. thaliana and $B$. rapa, including roots, stems, leaves, flowers and siliques, the expression patterns of all genes were investigated (Figure S8, Supplementary Tables 5, 6). BrCDPK-SnRK genes were found to be expressed in roots (99 BrCDPK-SnRKs; 77.95\%), stems (104; $81.89 \%)$, siliques $(106 ; 83.46 \%)$, leaves $(101 ; 79.53 \%)$, and flowers (119; 93.7\%) (Figures 8A-D). A total of 75 (88\%) AtCDPKSnRKs showed high expression (mean-normalized value $>1$ ) in at least one of the five tissues (Figure S8), including roots (34 AtCDPK-SnRKs; 40.00\%), stems (36; 42.35\%), siliques (22; 25.88\%), leaves $(19 ; 22.35 \%)$, and flowers (16; 18.23\%). Among the $116 \mathrm{CPKs}$ (including $46 \mathrm{AtCPKs}$ and $70 \mathrm{BrCDPKs}$ ), 4 (CPK28, CPK29, СPK30, and CPK33) were not expressed and 2 ( $B r C P K 4$ and $B r C P K 25)$ were only expressed in flower tissue (Figures 8A,B). In addition, a total of $34 \mathrm{BrCPKs}, 8$ BrCRKs, 4 BrPEPRKs, and 2 BrPPCKs had high expression levels (FPKM value $>10)$ in at least one tissue; $13 \mathrm{BrCPKs}$, 2 BrCRKs, and 1 BrPEPRK were highly expressed in all 5 tissues. However, only 2 SnRK genes were expressed in one tissue; the remains 93 SnRK genes were expressed in five tissues.

Subsequently, we selected the expression patterns of genes in five tissues on the phylogenetic tree of all CDPK-SnRK genes to investigate whether the functions of homologous genes were divergent (Figure S8). All CPKI, PEPRK, 


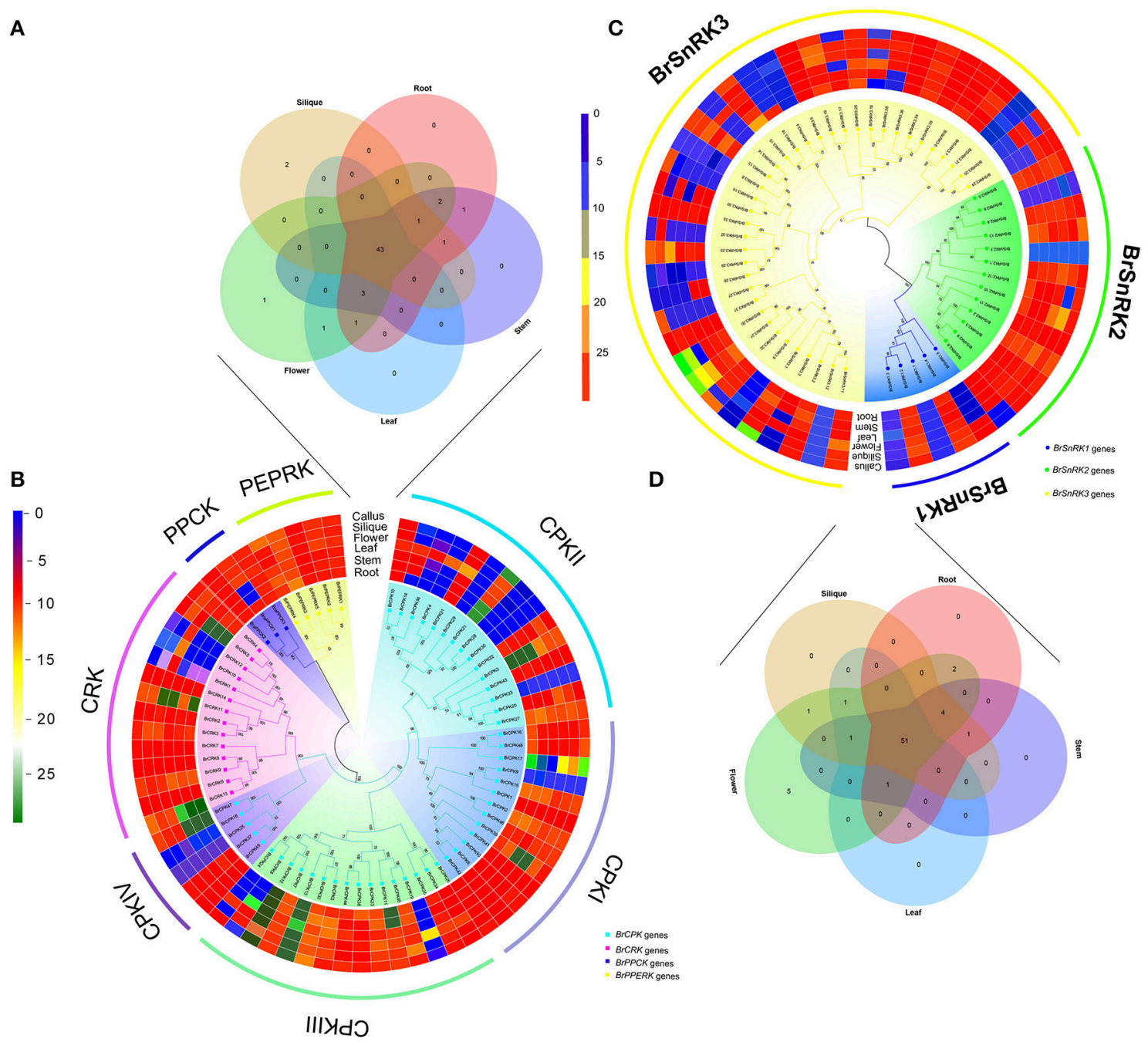

FIGURE 8 | Analysis of the CDPK-SnRK Genes in different tissues of Brassica rapa. (A,B) Heat map representation and hierarchical clustering of BrCPK, BrCRK, BrPEPRK, and BrPPCK genes in root, stem, leaf, flower, silique, and callus; Venn diagram depicting the distribution of shared expression of the BrCPK, BrCRK, BrPEPRK, and BrPPCK genes among five Brassica rapa tissues. (C,D) Heat map representation and hierarchical clustering of BrSnRK genes in root, stem, leaf, flower, silique, and callus; Venn diagram depicting the distribution of shared expression of the BrSnRK genes among five Brassica rapa tissues.

and PPCK and most CPKIII and CRK genes had high expression levels, suggesting significant roles of these genes in plant development. Most BrSnRK3s exhibited little or no expression. However, the AtSnRK3s were all expressed in five tissues, indicating that $\mathrm{BrSnRK} 3$ genes may have lost some functions after the duplication events (Figure S8).

\section{Expression Divergence and Coregulatory Networks of CDPK-SnRK Genes under Multiple Treatments in Brassica rapa}

CDPK-SnRKs are suggested to play an important role in the regulation of gene expression in response to abiotic stresses (Figure 9, Supplementary Table 7). To investigate the divergence information of the BrCDPK-SnRK gene family, the expression patterns following different treatments, including ABA, GA, $\mathrm{NaCl}$, heat, cold, and PEG treatments, were analyzed (Figures 9, 10). The qRT-PCR results demonstrate that those BrCDPKSnRK genes respond differentially to particular abiotic stresses. Sixteen percent of investigated BrCPK genes show increased expression levels upon GA at $6 \mathrm{~h}$, while the other $84 \%$ of genes display downregulation or no significant changes. Meanwhile, $20 \%$ of genes were upregulated at $1 \mathrm{~h}$ and $6 \mathrm{~h}$ under GA treatment (Figures 9A,C). Two genes (BrCPK4 and $B r C P K 10)$ were induced under both ABA and GA treatment (Figure 9B). During four abiotic stress treatments, excluding PEG, $80 \%$ of $\mathrm{BrCPK}$ genes were highly responsive to cold, $\mathrm{NaCl}$, and heat. We found that, with the exceptions of BrCPK2, 29, 23 and 26, all BrCPK genes were significantly induced in response to $\mathrm{NaCl}$ treatment (Figures 9D-F,H). Under heat treatment, we found that only two genes (BrCPK4 and $B r C P K 10)$ had 


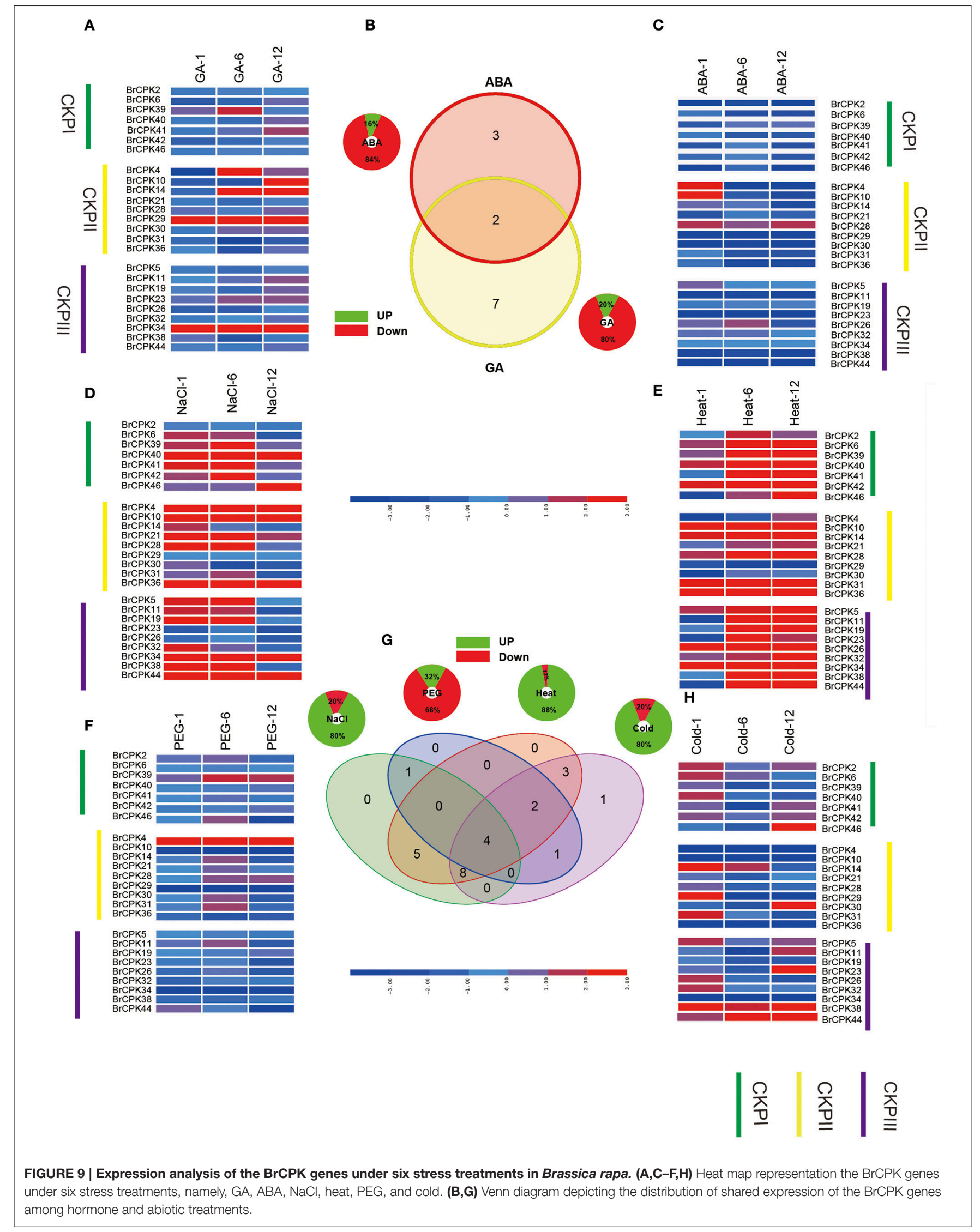




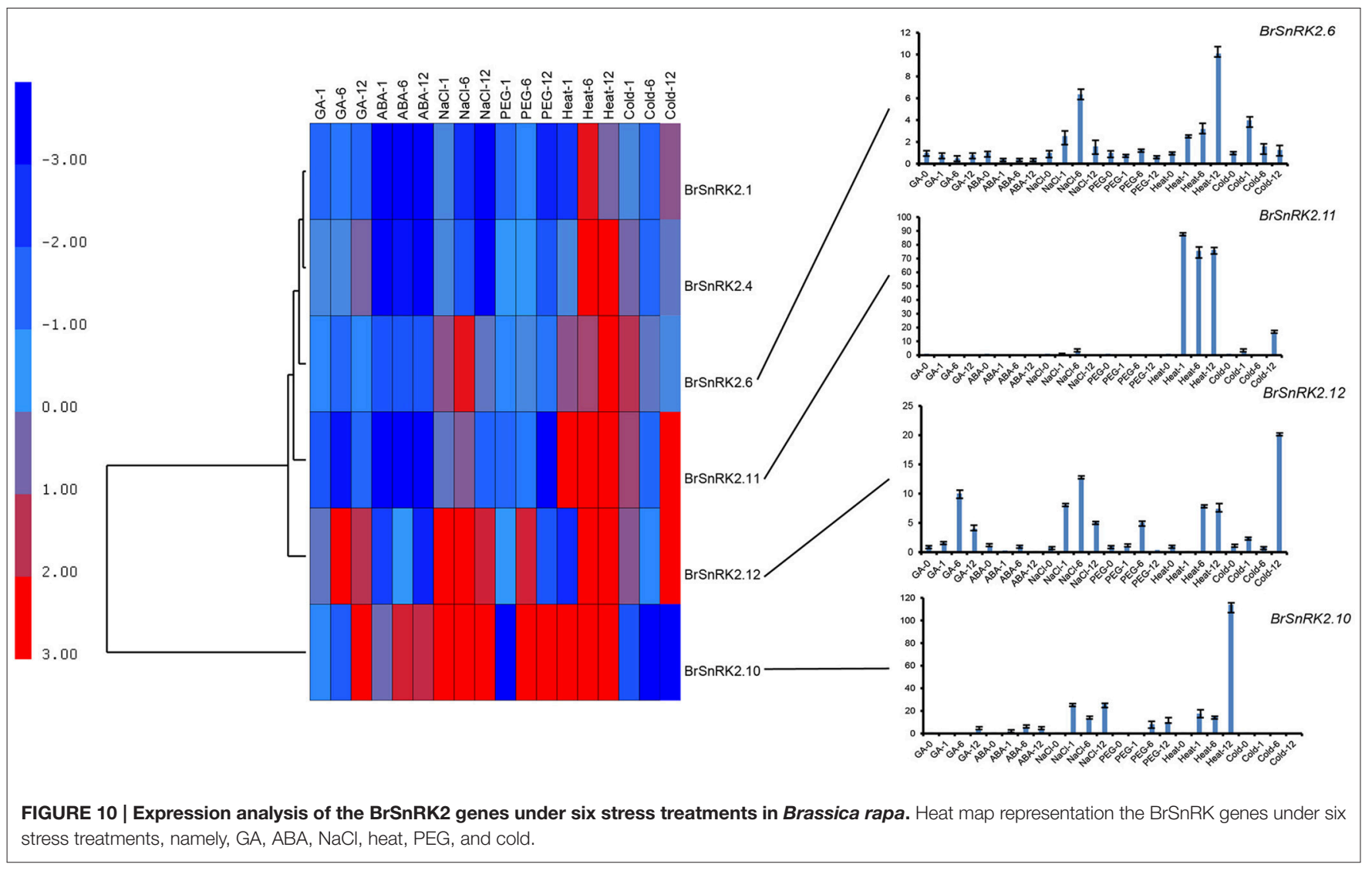

no expression; other $\mathrm{BrCPK}$ genes were highly expressed in $\mathrm{NaCl}$ treatment. SnRK2 genes recognized as coding for enzymes involved in abiotic stress signal transduction in plants (Kulik et al., 2011). Therefore, we selected six BrSnRK2 genes; all of these genes had the highest expression under heat treatment (Figure 10). Furthermore, BrSnRK2.12 was induced under all stress treatments (Figure 10).

To investigate the connections between these genes, coregulatory networks were established based on the PCCs of stress-inducible BrCDPK-SnRK gene pairs (Supplementary Table 8, Figure 11). All the BrCDPK-SnRKs appeared to have different degrees of positive correlation. Next, 25 BrCPKs with PCC values that were significant at the 0.05 significance level and were greater than 0.8 were collected and visualized to construct hormone and abiotic stress coregulatory networks (Figure 11A). Six BrSnRKs and seven BrCPKs had positive significant correlations (Figure 11C). Most correlations occurred among members belonging to the same group, suggesting that the gene duplication not only led to functional divergence but also enhanced the cooperative interaction of homologs to help plants to adapt to their complex environment. In addition, based on predicted Chinese cabbage BrCDPK-SnRK superfamily protein interactions, their interactions form a complex network in both Arabidopsis thaliana and B. rapa. Moreover, to study the protein interactions of stress response genes between $B$. rapa and Arabidopsis, STRING 9.1 was utilized (Figure S9). Figure S9 shows four complex interaction networks of CDPK-SnRKs, providing an overall view of the relationship between $B$. rapa and Arabidopsis.

\section{DISCUSSION}

In eukaryotes, protein kinases are involved in regulating key aspects of cellular function, including cell division, metabolism, and responses to external signals. CDPK-SnRK plays an important role in stress signal transduction in plants, such as wounding, salt or drought stress (Botella et al., 1996; Patharkar and Cushman, 2000; Saijo et al., 2000), cold (Monroy and Dhindsa, 1995; Saijo et al., 2000), hormone treatment (Abo-ElSaad and Wu, 1995; Botella et al., 1996; Davletova et al., 2001), light (Frattini et al., 1999), and pathogens (Romeis et al., 2001; Murillo et al., 2002).

The gene balance hypothesis predicts that genes whose products participate in signaling networks or macromolecular complexes or are transcription factors are more likely to be retained (Birchler and Veitia, 2007; Aad et al., 2012). In this study, we identified $49 \mathrm{BrCPKs}, 14 \mathrm{BrCRKs}, 3 \mathrm{BrPPCKs}, 5$ $B r P E P R K s$, and $56 \mathrm{BrSnRKs}$ in the B. rapa genome, and they contained a higher rate of copies than the $B$. rapa whole-genome level. This result suggests that these genes had a high degree of retention following WGD. By comparing the number of different duplicated types, gene copies, and distribution of the three subgenomes, we found that all the AtCRK, AtPEPRK, and 
A

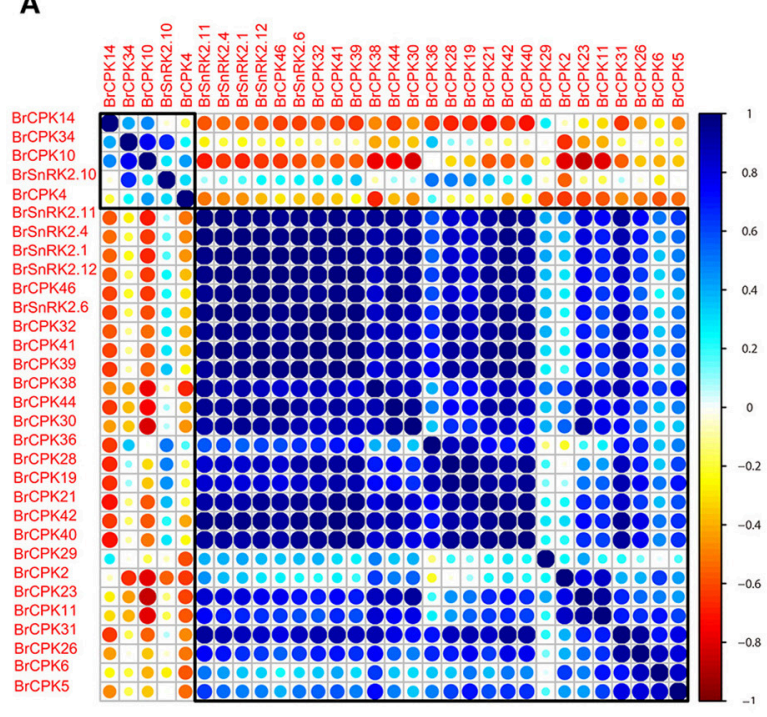

B

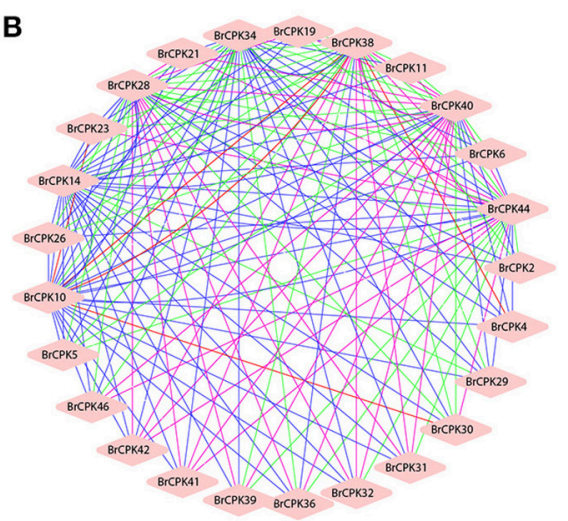

C

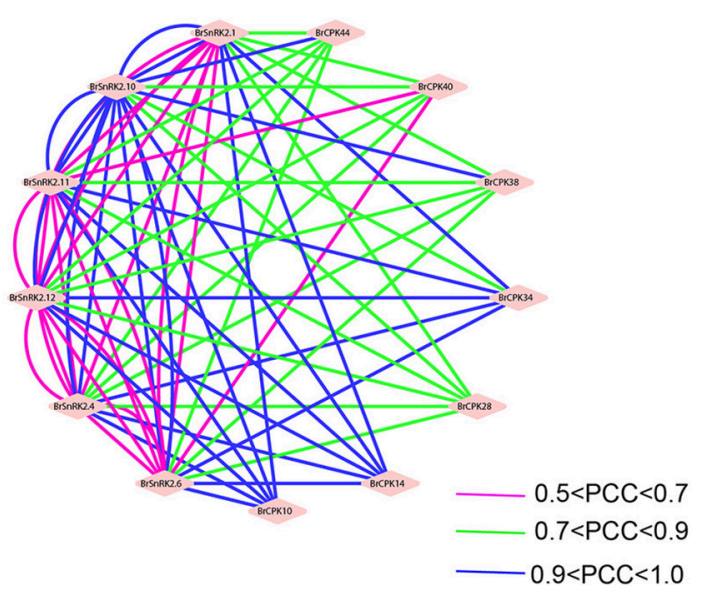

FIGURE 11 | Correlations and co-regulatory networks of 31 BrCDPK-SnRK genes under stress treatments. (A) Correlation analysis using the R package program. Each correlation is shown by the shades of blue and orange and the size of the circle shape. Blue and orange indicate a positive correlation and negative correlation, respectively. (B,C) Co-regulatory networks. The co-regulatory networks of 31 BrCDPK-SnRK genes under stress treatments were established based on the Pearson correlation coefficients (PCCs) of these gene pairs using transformed APCR data. The PCC of co-regulatory gene pairs was considered significant at the 0.05 significance level ( $p$-value), and different line colors and styles indicate the different significance levels of the co-regulated gene pairs.

AtPPCK orthologs were retained in B. rapa. In contrast, four AtCPK and two AtSnRK orthologs were completely lost. CRK, PEPRK, and PPCK were more preferentially retained than CPK and SnRK. CPK (66\%) and SnRK (43\%) had more than two copies retained in B. rapa, and more $\mathrm{BrCPK}(74 \%)$ and $\mathrm{BrSnRK}(70 \%)$ genes experienced segmental duplication. These preferentially retained CDPK-SnRK genes may have more important functions. At the same time, the important functions have been proved in previously researches.

For example, CDPK-SnRKs have been shown to have important roles in various physiological processes, including plant growth and development and abiotic and biotic stress responses in plants. The large number and variety of protein kinases that either have an EF-hand calcium-binding domain in their structure (CPK and CCaMK) or interact with EF-hand proteins (CCaMK, CaMK, CRK, and SnRK3) provides plants with a huge potential for interpreting specific calcium signals and for eliciting specific physiological responses. The SnRK1 kinase plays an important role in carbon-nitrogen interactions (Halford and Paul, 2004; Li et al., 2010) and in the transcription regulation of gene expression. In the developing tuber of potato, the expression level of SnRK1 was higher, lower in stem and lowest in leaf. The experiment on potato provided evidence for SnRK1 to regulate the transcription (Man et al., 1997). SnRK1 influence starch biosynthesis via regulating the expression of sucrose synthase and the activity of AGPase. SnRK1 activity can respond to sucrose appropriately (Halford and Paul, 2004). Antisense gene expressions in different plant indicate that SnRK1 has very important roles in plant growth and development processes (Halford et al., 2003). The SnRK2s are about 140-160 amino acids shorter than the SnRK1s, averaging about $40 \mathrm{kD}$ in size, and have a characteristic patch of acidic amino acids in their C-terminal domains (Halford et al., 2000). SnRK2 genes, which are a significant part of the ABA signal pathway, are involved in many processes that help plants resistant environmental pressures (Wang et al., 2015).The variety is further amplified in the $S n R K 3$ subfamily, since these kinases may interact with more than one CBL/SCaBP (Guo et al., 2001; Manns et al., 2001; Shi and Eberhart, 2001). Gene expression patterns can provide important clues for gene function. Therefore, the expression 
patterns of CDPK-SnRK genes under stresses were identified based on qRT-PCR analysis. Overall, expression patterns of CDPK-SnRK genes are dynamic in different tissues during different developmental stages and in response to abiotic stresses, demonstrating their regulatory roles in various cellular processes in B. rapa. This finding is consistent with the gene dosage hypothesis.

During evolutionary history, all extant angiosperms genome have undergone at least one and often multiple polyploidization events (Edger and Pires, 2009; Jiao et al., 2011a; Ohno, 2013). Brassica rapa experienced a complex WGD history, including $\gamma, \alpha$, and $\beta$ events, and an additional WGT event, providing an excellent chance to study the relationship between gene family fractionation and changes in plant morphotypes (Wang et al., 2011; Cheng et al., 2013). An extensive phylogenetic analysis revealed the evolutionary history of those CDPK-SnRK genes in B. rapa and in other plants. In total, 127 CDPKSnRK genes were grouped in seven classes in B. rapa. In addition, we identified CDPK-SnRK genes in 15 other plant species representing the major clades of terrestrial plants. These phylogenetic studies suggest that those identified CPK and SnRK genes were highly conserved in each class across a wide spectrum of plants, indicating their essential regulatory roles in the plant kingdom. For the CPK gene family, the number of genes grouped into each class, gene number and genome size are summarized in Figure 1. Furthermore, there is a difference among the five species in class I, II, III and IV in terms of gene numbers. It suggests that those four classes underwent extensive expansion in angiosperms. Class III was entirely lost during evolution in $V$. vinifera. For the SnRK gene family, two SnRK1 genes were found in V. carteri, suggesting that expansion of the SnRK gene family occurred after the divergence of green algae. Meanwhile, SnRK1, SnRK2, and SnRK3 were detected in Physcomitrella patens, indicating that the SnRK2 and SnRK3 gene subfamilies appear to be unique to plants, which consist with previously research (Hrabak et al., 2003). Overall, for the six species investigated, the number of CPK and SnRK genes remains different, indicating different evolutionary histories accompanied by rounds of WGD and subsequent gene losses/gains by natural selection constraints.

Most land plants have undergone polyploidization that led to WGD and provided opportunities for duplicated genes to diverge in different evolutionary ways. Each of these genes subsequently experienced one of three fates: subfunctionalization, neofunctionalization, or non-functionalization (deletion or pseudogenization). These fates provided opportunities for duplicated genes to gain functional diversification, resulting in more complex organisms. The $\mathrm{Ka} / \mathrm{Ks}$ substitution rate ratio is an indicator of the selection history on genes or gene regions. Commonly, if the value of $K a / K s$ is lower than 1 , the duplicated gene pairs may have evolved from purifying selection (also called as negative selection); $K a / K s=1$ means neutral selection, while $K a / K s>1$ means positive selection. In this study, the $K a / K s$ values for the duplicated gene pairs were small $(<0.01)$; thus, it is likely that these pairs have been under purifying selection. Neofunctionalization is an adaptive process during which one copy of a duplicated gene mutates to adopt a novel function that cannot be performed by the ancestral sequence. This mechanism can lead to the retention of both copies over long periods of time. In addition, by comparing the tissue expression pattern among the CDPK-SnRK genes in B. rapa and At. thaliana, we found that most of the duplicated genes maintained similar expression patterns, but some of the duplicated CDPK-SnRK genes demonstrated higher tissue specialization and diversification, also suggesting that CDPK-SnRKs underwent more neofunctionalization and subfunctionalization. In previous reports, even the most recently diverged paralogs differed in their catalytic efficiency, expression, and/or substrate spectrum, suggesting that duplicates have a relatively high rate of divergence in function.

In this study, we analyzed the evolutionary pattern, gene duplication, and expression divergence of the CDPK-SnRK genes in plants. We conducted a comparative evolutionary analysis with the genome information of selected plants, and our research provides new insight into the evolutionary history of the CDPK-SnRK gene family in plants. For example, CPK had a closer relationship with CRK, indicating that these two may share a common evolutionary origin; and PEPRK appeared between the salicoid duplication and the $\gamma$ event. By compared expression patterns among tissues, we found that the expansion of CDPK-SnRK genes seems to be associated with increasingly complex organs in the plants' evolution. Under different stress treatments, their coregulatory networks demonstrated that the CDPK-SnRK genes enhanced the cooperative interaction of homologs to adapt to the environment. In conclusion, this study provides useful resources for functional divergence and conservation in the CDPKSnRK gene superfamily and facilitates our understanding of the effect of polyploidy during the evolution of CDPK-SnRK genes.

\section{METHODS}

\section{Retrieval of Sequences}

The $B$. rapa sequences were downloaded from BRAD (http://brassicadb.org/brad/) (Wang et al., 2011). The Arabidopsis sequences were retrieved from TAIR (http://www.arabidopsis.org/), and the sequences of rice were retrieved from RGAP (http://rice.plantbiology.msu.edu/; Kawahara et al., 2013). The gene information on A. trichopoda genes was retrieved from the Amborella Genome Database (http://www.amborella.org/). The others 14 species' gene information was downloaded from Phytozome v9.1 (http:// www.phytozome.net/; Goodstein et al., 2012). The homologous CDPK-SnRK genes in other species were identified through comparison with Arabidopsis. First, BLASTP searches were performed against the rice protein sequences using an $E$-value threshold of $1 \times 10^{-10}$. The top-ranked rice hit was used for BLASTP searches of the Arabidopsis proteins to confirm homologies. Starting with both Arabidopsis and rice homologs, BLASTP searches were performed against the proteins of other species $\left(e<1 \times 10^{-10}\right.$, identity $\left.>70 \%\right)$. These potential sequences were analyzed using the tool SMART (http://smart. 
embl-heidelberg.de/) and the NCBI database (http://www.ncbi. nlm.nih.gov/).

\section{Identification of Gene Synteny and Duplicated CDPK-SnRK Genes}

BLAST and the Multiple Collinearity Scan toolkit (MCScanX) were used for gene synteny analysis according to previous reports (Wang et al., 2012). An all-against-all BLASTP comparison provided the pairwise gene information and the $P$-value for a primary clustering. Paired segments were extended by identifying the clustered genes using dynamic programming. The potentially duplicated genes were also identified using MCScanX. The positions of B. rapa CDPK-SnRK genes on the blocks were verified by searching for homologous genes between $A$. thaliana and three subgenomes of $B$. rapa (LF, MF1, and MF2) in BRAD (http://brassicadb.org/brad/searchSynteny.php; Wang et al., 2011). The syntenic diagram was drawn using Circos software (Krzywinski et al., 2009).

\section{Phylogenetic Analysis and Characterization of the CDPK-SnRK Gene Family}

Phylogenetic analyses were conducted using MEGA v5.073; maximum-likelihood (ML) trees were constructed with a bootstrap value of 1,000 replications to assess the reliability of the resulting trees. The genetic distance used in this study was also calculated with MEGA v5.0. To identify conserved motifs in the complete amino acid sequences of CDPK-SnRK proteins, we used MEME software (http://meme.sdsc.edu/meme/; Bailey et al., 2009). We researched the gene structure using Gene Structure Display (GSDS, http://gsds.cbi.pku.edu.cn/). The interaction network of CDPK-SnRK proteins in Chinese cabbage was constructed with STRING software (Search Tool for the Retrieval of Interacting Genes/Proteins, http://string-db.org/; Yamada et al., 2011).

\section{Calculation of the Ka/Ks and Dating of the Duplication Events}

The duplicated CDPK-SnRK genes from B. rapa were aligned using MUSCLE (Edgar, 2004). The protein alignments were translated into coding sequence alignments using an in-house Perl script. Ks (synonymous substitution rate) and $\mathrm{Ka}$ (nonsynonymous substitution rate) values were calculated based on the coding sequence alignments using the method of Nei and Gojobori as implemented in KaKs_calculator (Zhang et al., 2006). The $K s$ values of all duplicated $B r C D P K-$ SnRK genes were then plotted as the density and a boxplot using the $\mathrm{R}$ program (Ihaka and Gentleman, 1996). The divergence time was calculated with the formula $\mathrm{T}=K s / 2 r$, with $K s$ being the synonymous substitutions per site and $\mathrm{r}$ being the rate of divergence for nuclear genes from plants. The value of $\mathrm{r}$ was taken to be $1.5 \times 10^{-8}$ synonymous substitutions per site per year for dicotyledonous plants (Koch et al., 2000).

\section{Expression Pattern Analysis for CDPK-SnRK Genes}

For expression profiling of the CDPK-SnRK genes in $B$. rapa, we utilized the Illumina RNA-seq data that were previously generated and analyzed by Tong et al. (2013). Six tissues of B. rapa accession Chiifu-401-42, including callus, root, stem, leaf, flower, and silique, were analyzed. The transcript abundance was expressed as fragments per kilobase of exon model per million mapped reads (FPKM). The gene expression patterns of each tissue were analyzed using Cluster 3.0, and the expression values were $\log 2$ transformed. Finally, heat maps of hierarchical clustering were visualized using Tree View (http://jtreeview.sourceforge.net/). A. thaliana development expression profiling was analyzed using the AtGenExpress Visualization Tool (AVT; http://jsp.weigelworld. org/expviz/expviz.jsp) with mean-normalized values (Schmid et al., 2005). Venn diagrams were drawn using the $R$ program.

\section{Plant Material and Treatments}

Chinese cabbage (Chiifu-401-42) was used for the experiments. The germinated seeds were grown in plastic pots in a 3:1 soilvermiculite mixture, and the artificial growth conditions were set at $24 / 16^{\circ} \mathrm{C}$, with a photoperiod of $16 / 8 \mathrm{~h}$ for day/night and a relative humidity of $65-70 \%$. Four weeks later, seedlings were subjected to various treatments. For heat and cold treatment, the pots were exposed to $38^{\circ} \mathrm{C}$ or $4^{\circ} \mathrm{C}$; the other growth conditions were same as described earlier. Meanwhile, plants were cultured with the following four treatments: (1) control; (2) $100 \mu \mathrm{M} \mathrm{ABA}$; (3) $100 \mu \mathrm{M} \mathrm{GA}$; (4) (w/v) polyethylene glycol (PEG) 6,000, and (5) $250 \mathrm{mM} \mathrm{NaCl}$. All these treatments were performed over a continuous time course $(0,1,6,12 \mathrm{~h})$. Each treatment consisted of three biological replicates. All of the samples were frozen in liquid nitrogen and stored at $-70^{\circ} \mathrm{C}$ for RNA preparation.

\section{RNA Isolation and qRT-PCR Analyses}

Total RNA was isolated from treated frozen leaves using Trizol (Invitrogen, San Diego, CA, USA) according to the manufacturer's instructions. Specific primers used for qRTPCR were designed by Beacon Designer 7 and are shown in Supplementary Table 9. To check the specificity of the primers, we used BLAST against the Brassica genome. The qRT-PCR assays were performed with three biological and technical replicates. The reactions were performed using a StepOnePlus Real-Time PCR System (Applied Biosystems, Carlsbad, CA, USA). qRT-PCR was performed according to a previous report (Song et al., 2013). Relative fold expression changes were calculated using the comparative Ct-value method.

\section{Pearson Correlation Analyses}

Pearson correlation coefficients (PCCs) of stress-inducible CDPK-SnRK gene pairs were calculated using an in-house Perl script based on $\log 2$-transformed quantitative realtime (qRT)-PCR data (Tang et al., 2013). All gene pairs whose PCC was more than 0.8 and was significant at 
the 0.05 significance level ( $P$-value) were collected for a gene coregulatory network analysis. The coexpression networks were graphically visualized using Cytoscape based on the PCCs of these gene pairs (Shannon et al., 2003).

\section{AUTHOR CONTRIBUTIONS}

PW was responsible for the experimental design, data analysis, and manuscript writing. WD, WW, YL contributed to the experimental work. $\mathrm{XH}$ contributed to the interpretation of results and coordinated the study. All authors read and approved the final manuscript.

\section{REFERENCES}

Aad, G., Abajyan, T., Abbott, B., Abdallah, J., Khalek, S.A., Abdelalim, A., et al. (2012). Observation of a new particle in the search for the Standard Model Higgs boson with the ATLAS detector at the LHC. Phys. Lett. B 716, 1-29. doi: 10.1016/j.physletb.2012.08.020

Abo-El-Saad, M., and Wu, R. (1995). A rice membrane calcium-dependent protein kinase is induced by gibberellin. Plant Physiol. 108, 787-793. doi: 10.1104/pp.108.2.787

Albert, V. A., Barbazuk, W. B., Der, J. P., Leebens-Mack, J., Ma, H., Palmer, J. D., et al. (2013). The Amborella genome and the evolution of flowering plants. Science 342:1241089. doi: 10.1126/science.1241089

Alderson, A., Sabelli, P. A., Dickinson, J. R., Cole, D., Richardson, M., Kreis, M., et al. (1991). Complementation of snf1, a mutation affecting global regulation of carbon metabolism in yeast, by a plant protein kinase cDNA. Proc. Natl. Acad. Sci. U.S.A. 88, 8602-8605. doi: 10.1073/pnas.88.19.8602

Allen, G. J., Chu, S. P., Schumacher, K., Shimazaki, C. T., Vafeados, D., Kemper, A., et al. (2000). Alteration of stimulus-specific guard cell calcium oscillations and stomatal closing in Arabidopsis det3 mutant. Science 289, 2338-2342. doi: 10.1126 /science.289.5488.2338

Anderberg, R. J., and Walker-Simmons, M. (1992). Isolation of a wheat cDNA clone for an abscisic acid-inducible transcript with homology to protein kinases. Proc. Natl. Acad. Sci. U.S.A. 89, 10183-10187. doi: $10.1073 /$ pnas.89.21.10183

Asano, T., Kunieda, N., Omura, Y., Ibe, H., Kawasaki, T., Takano, M., et al. (2002). Rice SPK, a calmodulin-like domain protein kinase, is required for storage product accumulation during seed development phosphorylation of sucrose synthase is a possible factor. Plant Cell 14, 619-628. doi: 10.1105/tpc.010454

Asano, T., Tanaka, N., Yang, G., Hayashi, N., and Komatsu, S. (2005). Genomewide identification of the rice calcium-dependent protein kinase and its closely related kinase gene families: comprehensive analysis of the CDPKs gene family in rice. Plant Cell Physiol. 46, 356-366. doi: 10.1093/pcp/pci035

Assmann, S. M., and Wang, X.-Q. (2001). From milliseconds to millions of years: guard cells and environmental responses. Curr. Opin. Plant Biol. 4, 421-428. doi: 10.1016/S1369-5266(00)00195-3

Bailey, T. L., Boden, M., Buske, F. A., Frith, M., Grant, C. E., Clementi, L., et al. (2009). MEME SUITE: tools for motif discovery and searching. Nucleic Acids Res. 37, W202-W208. doi: 10.1093/nar/gkp335

Berridge, M. J., Lipp, P., and Bootman, M. D. (2000). The versatility and universality of calcium signalling. Nat. Rev. Mol. Biol. 1, 11-21. doi: $10.1038 / 35036035$

Birchler, J. A., and Veitia, R. A. (2007). The gene balance hypothesis: from classical genetics to modern genomics. Plant Cell 19, 395-402. doi: 10.1105/tpc.106.049338

Botella, J. R., Arteca, J. M., Somodevilla, M., and Arteca, R. N. (1996). Calciumdependent protein kinase gene expression in response to physical and chemical stimuli in mungbean (Vigna radiata). Plant Mol. Biol. 30, 1129-1137. doi: 10.1007/BF00019547

\section{ACKNOWLEDGMENTS}

This work was supported by the National Natural Science Foundation of China (No. 31330067, 31301782), the Science and Technology Pillar Program of Jiangsu Province (No. BE2013429), and the Agricultural Science and Technology Independent Innovation Funds of Jiangsu Province [CX(13)2006].

\section{SUPPLEMENTARY MATERIAL}

The Supplementary Material for this article can be found online at: http://journal.frontiersin.org/article/10.3389/fpls.2017. 00162/full\#supplementary-material

Bowers, J. E., Chapman, B. A., Rong, J., and Paterson, A. H. (2003). Unravelling angiosperm genome evolution by phylogenetic analysis of chromosomal duplication events. Nature 422, 433-438. doi: 10.1038/nature01521

Bush, D. S. (1995). Calcium regulation in plant cells and its role in signaling. Annu. Rev. Plant Biol. 46, 95-122. doi: 10.1146/annurev.pp.46.060195.000523

Cheng, F., Mandáková, T., Wu, J., Xie, Q., Lysak, M. A., and Wang, X. (2013). Deciphering the diploid ancestral genome of the mesohexaploid Brassica rapa. Plant Cell 25, 1541-1554. doi: 10.1105/tpc.113.110486

Cheng, S.-H., Willmann, M. R., Chen, H.-C., and Sheen, J. (2002). Calcium signaling through protein kinases. The Arabidopsis calcium-dependent protein kinase gene family. Plant Physiol. 129, 469-485. doi: 10.1104/pp.005645

Chico, J. M., Raıces, M., Téllez-Iñón, M. A. T., and Ulloa, R. M. A. (2002). A calcium-dependent protein kinase is systemically induced upon wounding in tomato plants. Plant Physiol. 128, 256-270. doi: 10.1104/pp.010649

Cho, K., Agrawal, G. K., Jwa, N.-S., Kubo, A., and Rakwal, R. (2009). Rice OsSIPK and its orthologs: a "central master switch" for stress responses. Biochem. Biophys. Res. Commun. 379, 649-653. doi: 10.1016/j.bbrc.2008.12.107

Choi, H.-I., Park, H.-J., Park, J. H., Kim, S., Im, M.-Y., Seo, H.-H., et al. (2005). Arabidopsis calcium-dependent protein kinase AtCPK32 interacts with $\mathrm{ABF} 4$, a transcriptional regulator of abscisic acid-responsive gene expression, and modulates its activity. Plant Physiol. 139, 1750-1761. doi: 10.1104/pp.105.069757

Chothia, C., Gough, J., Vogel, C., and Teichmann, S. A. (2003). Evolution of the protein repertoire. Science 300, 1701-1703. doi: 10.1126/science.1085371

D’Angelo, C., Weinl, S., Batistic, O., Pandey, G. K., Cheong, Y. H., Schültke, S., et al. (2006). Alternative complex formation of the Ca-regulated protein kinase CIPK1 controls abscisic acid-dependent and independent stress responses in Arabidopsis. Plant J. Cell Mol. Biol. 48, 857-872. doi: 10.1111/j.1365-313X.2006.02921.x

Davletova, S., Mészáros, T., Miskolczi, P., Oberschall, A., Török, K., Magyar, Z., et al. (2001). Auxin and heat shock activation of a novel member of the calmodulin like domain protein kinase gene family in cultured alfalfa cells. J. Exp. Bot. 52, 215-221. doi: 10.1093/jexbot/52.355.215

Edgar, R. C. (2004). MUSCLE: multiple sequence alignment with high accuracy and high throughput. Nucleic Acids Res. 32, 1792-1797. doi: 10.1093/nar/gkh340

Edger, P. P., and Pires, J. C. (2009). Gene and genome duplications: the impact of dosage-sensitivity on the fate of nuclear genes. Chromosome Res. 17, 699-717. doi: 10.1007/s10577-009-9055-9

Ehrhardt, D. W., Wais, R., and Long, S. R. (1996). Calcium spiking in plant root hairs responding to Rhizobium nodulation signals. Cell 85, 673-681. doi: 10.1016/S0092-8674(00)81234-9

Evans, N. H., McAinsh, M. R., and Hetherington, A. M. (2001). Calcium oscillations in higher plants. Curr. Opin. Plant Biol. 4, 415-420. doi: 10.1016/S1369-5266(00)00194-1

Fasano, J. M., Massa, G. D., and Gilroy, S. (2002). Ionic signaling in plant responses to gravity and touch. J. Plant Growth Regul. 21, 71-88. doi: $10.1007 / \mathrm{s} 003440010049$ 
Frattini, M., Morello, L., and Breviario, D. (1999). Rice calcium-dependent protein kinase isoforms OsCDPK2 and OsCDPK11 show different responses to light and different expression patterns during seed development. Plant Mol. Biol. 41, 753-764. doi: 10.1023/A:1006316422400

Fujii, H., Chinnusamy, V., Rodrigues, A., Rubio, S., Antoni, R., Park, S.-Y., et al. (2009). In vitro reconstitution of an abscisic acid signalling pathway. Nature 462, 660-664. doi: 10.1038/nature08599

Fujii, H., and Zhu, J.-K. (2009). Arabidopsis mutant deficient in 3 abscisic acid-activated protein kinases reveals critical roles in growth, reproduction, and stress. Proc. Natl. Acad. Sci. U.S.A. 106, 8380-8385. doi: 10.1073/pnas.0903144106

Gargantini, P. R., Gonzalez-Rizzo, S., Chinchilla, D., Raices, M., Giammaria, V., Ulloa, R. M., et al. (2006). A CDPK isoform participates in the regulation of nodule number in Medicago truncatula. Plant J. 48, 843-856. doi: 10.1111/j.1365-313X.2006.02910.x

Goodstein, D. M., Shu, S., Howson, R., Neupane, R., Hayes, R. D., Fazo, J., et al. (2012). Phytozome: a comparative platform for green plant genomics. Nucleic Acids Res. 40, D1178-D1186. doi: 10.1093/nar/gkr944

Guo, D., Chen, F., Wheeler, J., Winder, J., Selman, S., Peterson, M., et al. (2001). Improvement of in-rumen digestibility of alfalfa forage by genetic manipulation of lignin O-methyltransferases. Transgenic Res. 10, 457-464. doi: 10.1023/A:1012278106147

Halford, N. G., Boulyz, J. P., and Thomas, M. (2000). SNF1-related protein kinases (SnRKs)-regulators at the heart of the control of carbon metabolism and partitioning. Adv. Bot. Res. 32, 405-434. doi: 10.1016/S0065-2296(00)32031-6

Halford, N. G., and Hardie, D. G. (1998). SNF1-related protein kinases: global regulators of carbon metabolism in plants? Plant Mol. Biol. 37, 735-748. doi: 10.1023/A:1006024231305

Halford, N. G., Hey, S., Jhurreea, D., Laurie, S., McKibbin, R. S., Paul, M., et al. (2003). Metabolic signalling and carbon partitioning: role of Snf1related (SnRK1) protein kinase. J. Exp. Bot. 54, 467-475. doi: 10.1093/jxb/ $\operatorname{erg} 038$

Halford, N. G., and Paul, M. J. (2004). Highly conserved protein kinases involved in the regulation of carbon and amino acid metabolism. J. Exp. Bot. 55, 35-42. doi: $10.1093 / \mathrm{jxb} / \mathrm{erh} 019$

Hammond-Kosack, K. E., and Jones, J. (1996). Resistance gene-dependent plant defense responses. Plant Cell 8, 1773.

Harmon, A. C. (2003). Calcium-regulated protein kinases of plants. Gravit. Space Biol. Bull. 16, 83-90.

Harmon, A. C., Gribskov, M., Gubrium, E., and Harper, J. F. (2001). The CDPK superfamily of protein kinases. New Phytol. 151, 175-183. doi: 10.1046/j.1469-8137.2001.00171.x

Harmon, A. C., Gribskov, M., and Harper, J. F. (2000). CDPKs-a kinase for every $\mathrm{Ca}^{2+}$ signal? Trends Plant Sci. 5, 154-159. doi: 10.1016/S1360-1385(00)01577-6

Harper, J. F. (2001). Dissecting calcium oscillators in plant cells. Trends Plant Sci. 6, 395-397. doi: 10.1016/S1360-1385(01)02023-4

Holappa, L. D., and Walker-Simmons, M. (1995). The wheat abscisic acidresponsive protein kinase mRNA, PKABA1, is up-regulated by dehydration, cold temperature, and osmotic stress. Plant Physiol. 108, 1203-1210. doi: $10.1104 /$ pp.108.3.1203

Hrabak, E. M., Chan, C. W., Gribskov, M., Harper, J. F., Choi, J. H., Halford, N., et al. (2003). The Arabidopsis CDPK-SnRK superfamily of protein kinases. Plant Physiol. 132, 666-680. doi: 10.1104/pp.102.011999

Huang, Q.-S., Wang, H.-Y., Gao, P., Wang, G.-Y., and Xia, G.-X. (2008). Cloning and characterization of a calcium dependent protein kinase gene associated with cotton fiber development. Plant Cell Rep. 27, 1869-1875. doi: 10.1007/s00299-008-0603-0

Hwang, I., Sze, H., and Harper, J. F. (2000). A calcium-dependent protein kinase can inhibit a calmodulin-stimulated $\mathrm{Ca}^{2+}$ pump (ACA2) located in the endoplasmic reticulum of Arabidopsis. Proc. Natl. Acad. Sci. U.S.A. 97, 6224-6229. doi: 10.1073/pnas.97.11.6224

Ihaka, R., and Gentleman, R. (1996). R: a language for data analysis and graphics. J. Comput. Graphic. Stat. 5, 299-314. doi: 10.1080/10618600.1996.10474713

Imamura, M., Takahashi, Y. T., Nakamura, N., Htwe, N. M. P. S., Zheng, S. H., Shimazaki, K. I., et al. (2008). Isolation and characterization of a cDNA coding cowpea (Vigna unguiculata (L) Walp.) calcineurin B-Tike protein-interacting protein kinase, VuCIPKJ. Plant Biotechnol. 25, 437-445. doi: $10.5511 /$ plantbiotechnology.25.437
Innan, H., and Kondrashov, F. (2010). The evolution of gene duplications: classifying and distinguishing between models. Nat. Rev. Genet. 11, 97-108. doi: $10.1038 / \mathrm{nrg} 2689$

Jiao, Y., Shi, C., Edil, B. H., de Wilde, R. F., Klimstra, D. S., Maitra, A., et al. (2011a). DAXX/ATRX, MEN1, and mTOR pathway genes are frequently altered in pancreatic neuroendocrine tumors. Science 331, 1199-1203. doi: 10.1126/science. 1200609

Jiao, Y., Wickett, N. J., Ayyampalayam, S., Chanderbali, A. S., Landherr, L., Ralph, P. E., et al. (2011b). Ancestral polyploidy in seed plants and angiosperms. Nature 473, 97-100. doi: 10.1038/nature09916

Jossier, M., Bouly, J. P., Meimoun, P., Arjmand, A., Lessard, P., Hawley, S., et al. (2009). SnRK1 (SNF1-related kinase 1) has a central role in sugar and ABA signalling in Arabidopsis thaliana. Plant J. Cell Mol. Biol. 59, 316-328. doi: 10.1111/j.1365-313X.2009.03871.x

Kawahara, Y., de la Bastide, M., Hamilton, J. P., Kanamori, H., McCombie, W. R., Ouyang, S., et al. (2013). Improvement of the Oryza sativa Nipponbare reference genome using next generation sequence and optical map data. Rice 6:4. doi: 10.1186/1939-8433-6-4

Kim, K. N., Cheong, Y. H., Gupta, R., and Luan, S. (2000). Interaction specificity of Arabidopsis calcineurin B-like calcium sensors and their target kinases. Plant Physiol. 124, 1844-1853. doi: 10.1104/pp.124. 4.1844

Kim, K. N., Lee, J. S., Han, H., Choi, S. A., Go, S. J., and Yoon, I. S. (2003). Isolation and characterization of a novel rice $\mathrm{Ca}^{2+}$-regulated protein kinase gene involved in responses to diverse signals including cold, light, cytokinins, sugars and salts. Plant Mol. Biol. 52, 1191-1202. doi: 10.1023/B:PLAN.0000004330.62660.a2

Kleinow, T., Bhalerao, R., Breuer, F., Umeda, M., Salchert, K., and Koncz, C. (2000). Functional identification of an Arabidopsis snf4 ortholog by screening for heterologous multicopy suppressors of snf4 deficiency in yeast. Plant J. 23, 115-122. doi: 10.1046/j.1365-313x.2000.00809.x

Knight, H., and Knight, M. R. (2001). Abiotic stress signalling pathways: specificity and cross-talk. Trends Plant Sci. 6, 262-267. doi: 10.1016/S1360-1385(01)01946-X

Knight, H., Trewavas, A. J., and Knight, M. R. (1996). Cold calcium signaling in Arabidopsis involves two cellular pools and a change in calcium signature after acclimation. Plant Cell 8, 489-503. doi: 10.1105/tpc.8.3.489

Koch, M. A., Haubold, B., and Mitchell-Olds, T. (2000). Comparative evolutionary analysis of chalcone synthase and alcohol dehydrogenase loci in Arabidopsis, Arabis, and related genera (Brassicaceae). Mol. Biol. Evol. 17, 1483-1498. doi: 10.1093/oxfordjournals.molbev.a026248

Komatsu, S., Yang, G., Khan, M., Onodera, H., Toki, S., and Yamaguchi, M. (2007). Over-expression of calcium-dependent protein kinase 13 and calreticulin interacting protein 1 confers cold tolerance on rice plants. Mol. Genet. Genomics 277, 713-723. doi: 10.1007/s00438-007-0220-6

Kong, X., Lv, W., Jiang, S., Zhang, D., Cai, G., Pan, J., et al. (2013). Genome-wide identification and expression analysis of calcium-dependent protein kinase in maize. BMC Genomics 14:433. doi: 10.1186/1471-2164-14-433

Krzywinski, M., Schein, J., Birol, I., Connors, J., Gascoyne, R., Horsman, D., et al. (2009). Circos: an information aesthetic for comparative genomics. Genome Res. 19, 1639-1645. doi: 10.1101/gr.092759.109

Kudla, J., Batistič, O., and Hashimoto, K. (2010). Calcium signals: the lead currency of plant information processing. Plant Cell 22, 541-563. doi: $10.1105 /$ tpc. 109.072686

Kulik, A., Wawer, I., Krzywińska, E., Bucholc, M., and Dobrowolska, G. (2011). SnRK2 protein kinases-key regulators of plant response to abiotic stresses. Omics 15, 859-872. doi: 10.1089/omi.2011.0091

Lee, T.-H., Tang, H., Wang, X., and Paterson, A. H. (2013). PGDD: a database of gene and genome duplication in plants. Nucleic Acids Res. 41, D1152-D1158. doi: $10.1093 / \mathrm{nar} / \mathrm{gks} 1104$

Li, G., Peng, F., Zhang, L., Shi, X., and Wang, Z. (2010). Cloning and characterization of a SnRK1-encoding gene from Malus hupehensis Rehd. and heterologous expression in tomato. Mol. Biol. Rep. 37, 947-954. doi: 10.1007/s11033-009-9734-9

Li, A.-L., Zhu, Y.-F., Tan, X.-M., Wang, X., Wei, B., Guo, H.-Z., et al. (2008). Evolutionary and functional study of the CDPK gene family in wheat (Triticum aestivum L.). Plant Mol. Biol. 66, 429-443. doi: 10.1007/s11103-0079281-5 
Li, J., Wang, X.-Q., Watson, M. B., and Assmann, S. M. (2000). Regulation of abscisic acid-induced stomatal closure and anion channels by guard cell AAPK kinase. Science 287, 300-303. doi: 10.1126/science.287.5451.300

Llop-Tous, I., Domínguez-Puigjaner, E., and Vendrell, M. (2002). Characterization of a strawberry cDNA clone homologous to calcium-dependent protein kinases that is expressed during fruit ripening and affected by low temperature. J. Exp. Bot. 53, 2283-2285. doi: 10.1093/jxb/erf103

Ma, S.-Y., and Wu, W.-H. (2007). AtCPK23 functions in Arabidopsis responses to drought and salt stresses. Plant Mol. Biol. 65, 511-518. doi: 10.1007/s11103-007-9187-2

Man, A. L., Purcell, P. C., Hannappel, U., and Halford, N. G. (1997). Potato SNF1-related protein kinase: molecular cloning, expression analysis and peptide kinase activity measurements. Plant Mol. Biol. 34, 31-43. doi: 10.1023/A:1005765719873

Mathieu, J., Yant, L. J., Mürdter, F., Küttner, F., and Schmid, M. (2009). Repression of flowering by the miR172 target SMZ. Plos Biol. 7:e1000148. doi: 10.1371/journal.pbio.1000148

McAinsh, M. R., Evans, N. H., Montgomery, L. T., and North, K. A. (2002). Calcium signalling in stomatal responses to pollutants. New Phytol. 153, 441-447. doi: 10.1046/j.0028-646X.2001.00336.x

Manns, M. P., McHutchison, J. G., Gordon, S. C., Rustgi, V. K., Shiffman, M., Reindollar, R., et al. (2001). Peginterferon alfa-2b plus ribavirin compared with interferon alfa- $2 \mathrm{~b}$ plus ribavirin for initial treatment of chronic hepatitis $\mathrm{C}$ : a randomised trial. Lancet 358, 958-965. doi: 10.1016/S0140-6736(01)06102-5

Monroy, A. F., and Dhindsa, R. S. (1995). Low-temperature signal transduction: induction of cold acclimation-specific genes of alfalfa by calcium at 25 degrees C. Plant Cell 7, 321-331. doi: 10.2307/3869854

Munemasa, S., Hossain, M. A., Nakamura, Y., Mori, I. C., and Murata, Y. (2011). The Arabidopsis calcium-dependent protein kinase, CPK6, functions as a positive regulator of methyl jasmonate signaling in guard cells. Plant Physiol. 155, 553-561. doi: 10.1104/pp.110.162750

Murata, Y., Pei, Z.-M., Mori, I. C., and Schroeder, J. (2001). Abscisic acid activation of plasma membrane $\mathrm{Ca}^{2+}$ channels in guard cells requires cytosolic NAD (P) $\mathrm{H}$ and is differentially disrupted upstream and downstream of reactive oxygen species production in abi1-1 and abi2-1 protein phosphatase $2 \mathrm{C}$ mutants. Plant Cell 13, 2513-2523. doi: 10.1105/tpc.13.11.2513

Murillo, M., Gamazo, C., Goñi, M., Irache, J. M., and Blanco-Prieto, M. (2002). Development of microparticles prepared by spray-drying as a vaccine delivery system against brucellosis. Int. J. Pharm. 242, 341-344. doi: 10.1016/S0378-5173(02)00212-0

Nakashima, K., Fujita, Y., Kanamori, N., Katagiri, T., Umezawa, T., Kidokoro, S., et al. (2009). Three Arabidopsis SnRK2 protein kinases, SRK2D/SnRK2. 2, SRK2E/SnRK2. 6/OST1 and SRK2I/SnRK2. 3, involved in ABA signaling are essential for the control of seed development and dormancy. Plant Cell Physiol. 50, 1345-1363. doi: 10.1093/pcp/pcp083

Ohno, S. (2013). Evolution by Gene Duplication. London; Berlin; Heidelberg; New York, NY: George Alien \& Unwin Ltd.; Springer-Verlag.

Ohno, S., Wolf, U., and Atkin, N. B. (1968). Evolution from fish to mammals by gene duplication. Hereditas 59, 169-187. doi: 10.1111/j.1601-5223.1968.tb02169.x

Pandey, G. K., Grant, J. J., Cheong, Y. H., Kim, B. G., Li, L. G., and Luan, S. (2008). Calcineurin-B-like protein CBL9 interacts with target kinase CIPK3 in the regulation of ABA response in seed germination. Mol. Plant 1, 238-248. doi: $10.1093 / \mathrm{mp} / \mathrm{ssn} 003$

Patharkar, O. R., and Cushman, J. C. (2000). A stress-induced calcium-dependent protein kinase from Mesembryanthemum crystallinum phosphorylates a two-component pseudo-response regulator. Plant J. 24, 679-691. doi: 10.1046/j.1365-313x.2000.00912.x

Pei, Z.-M., Murata, Y., Benning, G., Thomine, S., Klüsener, B., Allen, G. J., et al. (2000). Calcium channels activated by hydrogen peroxide mediate abscisic acid signalling in guard cells. Nature 406, 731-734. doi: 10.1038/35021067

Plieth, C., and Trewavas, A. J. (2002). Reorientation of seedlings in the earth's gravitational field induces cytosolic calcium transients. Plant Physiol. 129, 786-796. doi: 10.1104/pp.011007

Raíces, M., Ulloa, R. M., MacIntosh, G. C., Crespi, M., and Téllez-Iñón, M. T. (2003). StCDPK1 is expressed in potato stolon tips and is induced by high sucrose concentration. J. Exp. Bot. 54, 2589-2591. doi: 10.1093/jxb/ $\operatorname{erg} 282$
Ray, S., Agarwal, P., Arora, R., Kapoor, S., and Tyagi, A. K. (2007). Expression analysis of calcium-dependent protein kinase gene family during reproductive development and abiotic stress conditions in rice (Oryza sativa L. ssp. indica). Mol. Genet. Genomics 278, 493-505. doi: 10.1007/s00438-007-0267-4

Rensing, S. A. (2014). Gene duplication as a driver of plant morphogenetic evolution. Curr. Opin. Plant Biol. 17, 43-48. doi: 10.1016/j.pbi.2013.11.002

Ritchie, S. M., Swanson, S. J., and Gilroy, S. (2002). From common signalling components to cell specific responses: insights from the cereal aleurone. Physiol. Plant. 115, 342-351. doi: 10.1034/j.1399-3054.2002.1150303.x

Romeis, T., Ludwig, A. A., Martin, R., and Jones, J. D. (2001). Calcium-dependent protein kinases play an essential role in a plant defence response. EMBO J. 20, 5556-5567. doi: 10.1093/emboj/20.20.5556

Romeis, T., Piedras, P., and Jones, J. D. (2000). Resistance gene-dependent activation of a calcium-dependent protein kinase in the plant defense response. Plant Cell 12, 803-815. doi: 10.1105/tpc.12.5.803

Rudd, J. J., and Franklin-Tong, V. E. (2001). Unravelling response-specificity in $\mathrm{Ca}^{2+}$ signalling pathways in plant cells. New Phytol. 151, 7-33. doi: 10.1046/j.1469-8137.2001.00173.x

Saijo, Y., Hata, S., Kyozuka, J., Shimamoto, K., and Izui, K. (2000). Over-expression of a single $\mathrm{Ca}+$-dependent protein kinase confers both cold and salt/drought tolerance on rice plants. Plant J. 23, 319-327. doi: 10.1046/j.1365-313x.2000.00787.x

Sanders, D., Brownlee, C., and Harper, J. F. (1999). Communicating with calcium. Plant Cell 11, 691-706. doi: 10.1105/tpc.11.4.691

Sanders, D., Pelloux, J., Brownlee, C., and Harper, J. F. (2002). Calcium at the crossroads of signaling. Plant Cell 14, S401-S417. doi: 10.1105/tpc.002899

Schmid, M., Davison, T. S., Henz, S. R., Pape, U. J., Demar, M., Vingron, M., et al. (2005). A gene expression map of Arabidopsis thaliana development. Nat. Genet. 37, 501-506. doi: 10.1038/ng1543

Shannon, P., Markiel, A., Ozier, O., Baliga, N. S., Wang, J. T., Ramage, D., et al. (2003). Cytoscape: a software environment for integrated models of biomolecular interaction networks. Genome Res. 13, 2498-2504. doi: 10.1101/gr.1239303

Shi, Y., and Eberhart, R. C. (2001). "Fuzzy adaptive particle swarm optimization," in Evolutionary Computation, Proceedings of the 2001 Congress on (Boston: IEEE), 101-106.

Song, X., Li, Y., and Hou, X. (2013). Genome-wide analysis of the AP2/ERF transcription factor superfamily in Chinese cabbage (Brassica rapa ssp. pekinensis). BMC Genomics 14:573. doi: 10.1186/1471-2164-14-573

Stebbins, C. Jr. (1950). Variation and Evolution in Plants. New York, NY: Columbia University Press.

Szczegielniak, J., Borkiewicz, L., Szurmak, B., Lewandowska-Gnatowska, E., Statkiewicz, M., Klimecka, M., et al. (2012). Maize calcium-dependent protein kinase (ZmCPK11): local and systemic response to wounding, regulation by touch and components of jasmonate signaling. Physiol. Plant. 146, 1-14. doi: 10.1111/j.1399-3054.2012.01587.x

Tang, J., Wang, F., Wang, Z., Huang, Z., Xiong, A., and Hou, X. (2013). Characterization and co-expression analysis of WRKY orthologs involved in responses to multiple abiotic stresses in Pak-choi (Brassica campestris ssp. chinensis). BMC Plant Biol. 13:188. doi: 10.1186/1471-2229-13-188

Taylor, L. P., and Hepler, P. K. (1997). Pollen germination and tube growth. Annu. Rev. Plant Biol. 48, 461-491. doi: 10.1146/annurev.arplant.48.1.461

Thuleau, P., Schroeder, J. I., and Ranjeva, R. (1998). Recent advances in the regulation of plant calcium channels: evidence for regulation by G-proteins, the cytoskeleton and second messengers. Curr. Opin. Plant Biol. 1, 424-427. doi: 10.1016/S1369-5266(98)80267-7

Tong, C., Wang, X., Yu, J., Wu, J., Li, W., Huang, J., et al. (2013). Comprehensive analysis of RNA-seq data reveals the complexity of the transcriptome in Brassica rapa. BMC Genomics 14:689. doi: 10.1186/1471-2164-14-689

Trewavas, A. J., and Malhó, R. (1998). $\mathrm{Ca}^{2+}$ signalling in plant cells: the big network! Curr. Opin. Plant Biol. 1, 428-433. doi: 10.1016/S1369-5266(98)80268-9

Tuskan, G. A., Difazio, S., Jansson, S., Bohlmann, J., Grigoriev, I., Hellsten, U., et al. (2006). The genome of black cottonwood, Populus trichocarpa (Torr. \& Gray). Science 313, 1596-1604. doi: 10.1126/science.1128691

Wang, C., Liu, Y., Li, S. S., and Han, G.-Z. (2015). Insights into the originand evolution of the plant hormone signaling machinery. Plant Physiol. 167, 872-886. doi: 10.1104/pp.114.247403 
Wang, X., Wang, H., Wang, J., Sun, R., Wu, J., Liu, S., et al. (2011). The genome of the mesopolyploid crop species Brassica rapa. Nat. Genet. 43, 1035-1039. doi: $10.1038 /$ ng.919

Wang, Y., Tang, H., DeBarry, J. D., Tan, X., Li, J., Wang, X., et al. (2012). MCScanX: a toolkit for detection and evolutionary analysis of gene synteny and collinearity. Nucleic Acids Res. 40, e49-e49. doi: 10.1093/nar/gkr1293

Yamada, T., Letunic, I., Okuda, S., Kanehisa, M., and Bork, P. (2011). iPath2. 0: interactive pathway explorer. Nucleic Acids Res. 39(Suppl. 2), W412-W415. doi: 10.1093/nar/gkr313

Zhang, Z., Li, J., Zhao, X.-Q., Wang, J., Wong, G. K.-S., and Yu, J. (2006). KaKs_Calculator: calculating $\mathrm{Ka}$ and Ks through model selection and model averaging. Genomics Proteomics Bioinformatics 4, 259-263. doi: 10.1016/S1672-0229(07)60007-2

Zuo, R., Hu, R., Chai, G., Xu, M., Qi, G., Kong, Y., et al. (2013). Genome-wide identification, classification, and expression analysis of CDPK and its closely related gene families in poplar (Populus trichocarpa). Mol. Biol. Rep. 40, 2645-2662. doi: 10.1007/s11033-0122351-z

Conflict of Interest Statement: The authors declare that the research was conducted in the absence of any commercial or financial relationships that could be construed as a potential conflict of interest.

Copyright (c) 2017 Wu, Wang, Duan, Li and Hou. This is an open-access article distributed under the terms of the Creative Commons Attribution License (CC $B Y)$. The use, distribution or reproduction in other forums is permitted, provided the original author(s) or licensor are credited and that the original publication in this journal is cited, in accordance with accepted academic practice. No use, distribution or reproduction is permitted which does not comply with these terms. 